\title{
Hybrid Input-output Conformance and Test Generation ${ }^{\star}$
}

\author{
Michiel van Osch \\ Technische Universiteit Eindhoven \\ Department of Mathematics and Computer Science \\ 5600 MB, Eindhoven, The Netherlands \\ m.p.w.j.van.osch@tue.nl
}

\begin{abstract}
Input-output conformance test theory for discrete systems has established itself in research and industry already. A couple of years ago also input-output conformance test theories for timed systems were defined. The next step is to develop conformance test theory for hybrid systems as well. In this report we present a conformance relation for model-based testing of hybrid systems and we formalize tests for hybrid systems.
\end{abstract}

\section{Introduction}

A hybrid system is a system with both discrete behavior and continuous behavior. It has discrete controllers or computers, running discrete software, with discrete input and discrete output; it receives continuous input through the observations of sensors; and it generates continuous output through actuators (e.g. motors, hydraulics, heaters). A hybrid system can be very complex and testing it thoroughly can be of critical importance. This is the case when the safety of people is involved or when an unreliable machine may cause a huge loss of profit for a manufacturer.

The main purpose of model-based conformance testing is to develop a test tool that uses a formal specification to generate tests. These tests describe the input with which the system under test should be stimulated, and the output that is expected from it. Model-based test generation is preferably supported by a mathematical input-output conformance theory that formally defines when an implementation is to be considered conform a specification. With respect to this theory, the set of tests generated from a specification by the algorithm can then be proved to be sound (meaning that only faulty implementations can fail a generated test) and exhaustive (meaning that every faulty implementation fails at least one generated test). Some of the main advantages of model-based testing are that, since tests are automatically generated and executed, many more tests

\footnotetext{
* This work has been carried out as part of the TANGRAM project under the responsibility of the Embedded Systems Institute. This project is partially supported by the Netherlands Ministry of Economic Affairs under grant TSIT2026.
} 
can be performed in less time, and tests can be easily repeated. Moreover, the specifications can also be used to formally verify the design of a system.

Tretmans [9] proposed an input-output conformance test theory for discrete event systems; it led to the test tool TorX [11], which has been successfully applied in several industrial settings. Recently, extensions for real-time systems $[2,6,7]$ and extensions for systems with data $[4,5]$ were proposed. In this report we present an input-output conformance test theory for hybrid systems.

We start from the assumption that the specification is a hybrid transition system, which has a discrete transition relation labelled with actions and a continuous transition relation labelled with trajectories (flows of variables). With every hybrid transition relation we associate a set of tests, which are themselves a special kind of hybrid transition systems. Roughly, tests are generated from the specification by a recursive algorithm that chooses to either select an input action or an input trajectory from the specification, or to evaluate the observed output (actions and trajectories). If the observed output action or trajectory is allowed according to the specification, test case generation may continue or end with the verdict pass, whereas if the output action or trajectory is not allowed according to the specification, then the test aborts with the verdict fail.

For the purpose of validating the test generation algorithm, we also develop a mathematical input-output conformance theory for hybrid transition systems. In this theory it is assumed that also the implementation is a hybrid transition system, which is moreover assumed to be input enabled (at all times it should be able to accept any input from its environment). We propose an input-output conformance relation that formalizes the idea that, in every reachable state, a correct implementation should only perform output actions or output flows that are allowed according to the specification.

This report is organized as follows. In Section 2 we informally discuss some of the issues that play a rôle in a theory of model-based input-output conformance testing for hybrid systems. In Section 3 we briefly review the ioco theory of Tretmans. In Section 4 we introduce hybrid transition systems. In Section 5 we formalize when an implementation (an input enabled hybrid transition system) should be considered conform a specification (also a hybrid transition system). In Section 6 we inductively associate with every specification a set of tests. In Section 8 we prove soundness and exhaustiveness of our tests. In Section 8 we discuss some issues that will still need to be solved before our theory can be implemented. The report ends with some conclusions in Section 9.

\section{Hybrid Model-based Testing by an Example}

In this section we informally introduce the main concepts of model-based testing for hybrid systems. Consider a simple brake control system of a car. The system allows a car to stay behind another car. The system continuously measures the distance with the car in front. If the car comes too close to the car in front it starts braking. The brake system can be turned ON or turned OFF. If the 
system is turned OFF, then a warning light is turned ON to notify the driver. If the system detects a new car in front, the driver is also notified.

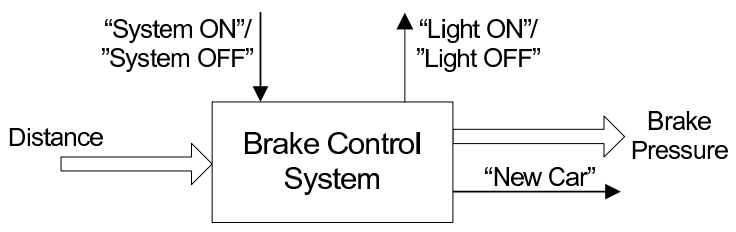

Fig. 1. A Braking System of a Car

Testing a system means stimulating it with input behavior and observing the output behavior. For instance, the brake system is stimulated by turning it ON and it is observed whether the warning light is turned OFF. Or, the sensor which is measuring the distance with the car in front is stimulated with distance measurement and the change of brake pressure is observed.

What makes testing hybrid systems difficult is that continuous input and continuous output always occur, that they occur in synchrony and that they depend on each other. If the brake system is stimulated with a steadily decreasing distance, then the brake pressure should increases steadily. It is even possible that the continuous input depends on the continuous output. For instance, if the car brakes, the amount of brake pressure influences how rapidly the distance with the car in front decreases (or increases). Furthermore, discrete output behavior also depends on continuous input behavior and discrete output behavior may have time constraints. For instance, if the distance measurement with the car in front makes a jump (which means a new car is detected), then a "New Car" output has to occur within 0.5 seconds. A test is passed if only expected output is observed (given the input applied) and it fails if an unexpected output is observed. If the brake pressure does not increase while the distance with the car in front decreases (as expected), then the test fails.

The goal of model-based testing, in the form we consider it, is to automate test generation and execution. The behavior of the system is specified by a formal model and tests are automatically generated from this specification. The specification can be a transition system, an automaton, a process algebra term, or a (formal) specification language. Tests are generated by selecting discrete or continuous input from the specification and enumerating the possible observations. A verdict pass or fail is attached to each possible observation in accordance with the specification. Tests are executed by automatically stimulating the implementation with the input described by the test and simultaneously observing the output from the implementation.

It is usually assumed that the implementation is input enabled. It is possible to stimulate the system under test (which is the system that is tested) with every conceivable behavior at any moment in time.This assumption simplifies 
automated test generation and execution because the test tool does not need to check whether the implementation accepts the input. In most cases it is also a natural assumption on the implementation. Stimulating the brake system with "Hello World" instead of "System ON" affects the system and it should be possible to test this. It is not required that the specification is input enabled. This makes it possible to steer the test process as only the behavior is tested for which the specification provides the input. For example it is possible to only specify gradual distance change and test the system using this input, or it is possible to specify a jump in distance change and test the system using this input.

The conformance relation formally defines if an implementation conforms to the specification. The test generation procedure defines how tests are generated from a specification. With a formal definition of how a test is constructed and a formal conformance relation it is possible to prove whether our tests are sound and exhaustive with respect to the conformance relation. That is, if the implementation conforms to the specification then the implementation will pass all tests that can be generated from the specification and if the implementation is not conform the specification then it is possible to generate a test which fails.

In this report we define a formal conformance relation and formal tests for hybrid systems. Before this, we first review the input-output conformance theory of Tretmans.

\section{Input-Output Conformance and Test Generation for Discrete Systems}

The input-output conformance relation (ioco) as defined by Tretmans $[9,10]$ is a relation between a specification and an implementation. An implementation is considered input-output conform a specification if in every (set of) states the system can be in after executing a trace, the set of output actions possible by the implementation is a subset of the set of output actions possible by the specification. This relation is useful in testing. It defines that when we detect an action of an implementation that is not specified, the implementation is not (ioco) correct. In other words, it defines when we consider an implementation to contain mistakes.

Fig. 2 depicts two models of a coffee machine. In Model 1 the coffee machine accepts a coin (input) and produces coffee (output), after which a coin can be accepted again. In Model 2 the coffee machine accepts a coin and produces nondeterministically coffee or tea. Model 1 coforms to Model 2. In both models coffee can be produced after accepting a coin. However, Model 2 does not conform to Model 1. Model 2 can produce tea also as output after a coin, while that is not allowed according to Model 1.

In traditional ioco theory the specification is assumed to be by a labelled transition systems (LTS) and the implementation is assumed to be an inputoutput transition system (IOTS).

Definition 1. A labelled transition system is a tuple $\mathcal{L}=\left(S, s_{0}, A, \rightarrow\right)$, where: 


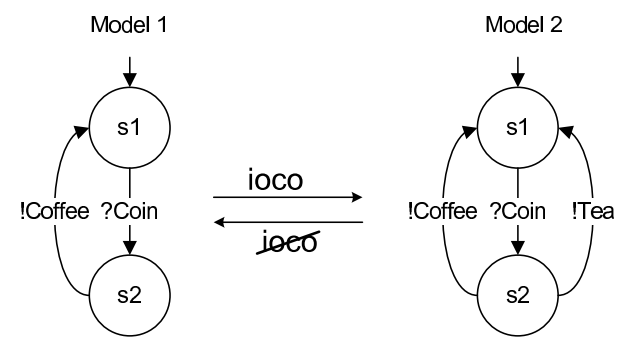

Fig. 2. Example Transition Systems

- $S$ is a (possibly infinite) set of states;

$-s_{0} \in S$ is the initial state;

- $A$ is a (possibly infinite) set of action labels. The special label $\tau \notin A$ is the unobservable action. We write $A_{\tau}$ for $A \cup\{\tau\}$;

$-\rightarrow \subseteq S \times A_{\tau} \times S$ is the transition relation. We write $s \stackrel{\mu}{\rightarrow} s^{\prime}$ for $\left(s, \mu, s^{\prime}\right) \in \rightarrow$.

Definition 2. An input-output transition system is a labelled transition system in which the set of action labels is partitioned into a set of input actions $A_{I}$ and a set of output actions $A_{O}$. An IOTS is required to be input-enabled in all states, i.e., every input is possible in every state: for all $s \in S$ and $a \in A_{I}: s \stackrel{a}{\rightarrow}$.

The generalized transition relation $\Rightarrow \subseteq S \times A^{*} \times S$ is definied by:

- $s \stackrel{\epsilon}{\Rightarrow} s$

- if $s \stackrel{\alpha}{\Rightarrow} s^{\prime \prime}$ and $s^{\prime \prime} \stackrel{\tau}{\rightarrow} s^{\prime}$ then $s \stackrel{\alpha}{\Rightarrow} s^{\prime}$;

- if $s \stackrel{\alpha}{\Rightarrow} s^{\prime \prime}$ and $s^{\prime \prime} \stackrel{\mu}{\longrightarrow} s^{\prime}$ and $\mu \neq \tau$ then $s \stackrel{\alpha \mu}{\Rightarrow} s^{\prime}$.

Furthermore, $s \stackrel{\mu}{\rightarrow}$ denotes there is a state $s^{\prime}$ such that $s \stackrel{\mu}{\longrightarrow} s^{\prime}$, and $s \stackrel{\alpha}{\Rightarrow}$ denotes there is a state $s^{\prime}$ such that $s \stackrel{\alpha}{\Rightarrow} s^{\prime}$.

A powerful concept in ioco is the notion of quiescence. A quiescent state is a state in which no output is possible and is never possible without providing input first.

Definition 3. Let $\mathcal{L}=\left(S, s_{0}, A_{I} \cup A_{O}, \rightarrow\right)$ be an LTS, then a state $s \in S$ is quiescent, denoted by $\delta(s)$, if for all $\mu \in A_{O} \cup\{\tau\}: s \stackrel{\mu}{\leftrightarrow}$.

This notion is important because an implementation that cannot produce an output, while according to the specification it should, is also not conform that specification. For the conformance relation a new label $\delta$ is introduced, which is not in the set $A$. From now on, $A \cup\{\delta\}$ is abbreviated as $A_{\delta}$.

In order to define the ioco relation the following definitions are needed:

Definition 4. Let $\mathcal{L}=\left(S, s_{0}, A_{\delta \tau}, \rightarrow\right)$ be an LTS with $A_{\delta \tau}=A_{I} \cup A_{O} \cup\{\delta, \tau\}$ and let $s \in S$ be a state; then 
- Straces $(\mathcal{L})=\left\{\alpha \in\left(A_{\delta}\right)^{*} \mid s_{0} \stackrel{\alpha}{\Rightarrow} \delta\right\}$ is the set of all suspension traces. Here, $\Rightarrow_{\delta} \subseteq S \times A_{\delta}^{*} \times S$ is defined by the following rules:

- if $s \stackrel{\alpha}{\Rightarrow} s^{\prime}$, then $s \stackrel{\alpha}{\Rightarrow} \delta s^{\prime}$;

- if $\delta(s)$, then $s \stackrel{\delta}{\Rightarrow} \delta$;

- if $s \stackrel{\alpha}{\Rightarrow} \delta s^{\prime \prime}$ and $s^{\prime \prime} \stackrel{\mu}{\Rightarrow} \delta s^{\prime}$, then $\stackrel{\alpha \mu}{\Rightarrow} \delta s^{\prime}$.

- $s$ after $\alpha=\left\{s^{\prime} \mid s \stackrel{\alpha}{\Rightarrow}_{\delta} s^{\prime}\right\}$ is the set of states after trace $\alpha$, with start state $s$.

- $C$ after $\alpha=\bigcup_{s \in C} s$ after $\alpha$, with $C \subseteq S$.

- $\operatorname{out}(s)= \begin{cases}\{\delta\} & \text { if } \delta(s) ; \text { and } \\ \left\{\mu \in A_{O} \mid s \stackrel{\mu}{\rightarrow}\right\} & \text { otherwise, }\end{cases}$

is the set of output transition labels from a state $s$.

- out $(C)=\bigcup_{c \in C} \operatorname{out}(c)$ with $C \subseteq S$.

An implementation is input-output conform a specification if for all suspension traces in the specification, the set of outputs possible after that trace is a subset of the set of outputs possible in the implementation. I.e. an implementation is not conform a specification when the implementation does an output action that was not specified.

Definition 5. Let $\mathcal{I}$ be an IOTS and let $\mathcal{S}$ be a LTS, then the binary relation ioco is defined as:

$\mathcal{I}$ ioco $\mathcal{S} \Longleftrightarrow \forall_{\alpha \in \operatorname{Straces}(\mathcal{S})}: \operatorname{out}(\mathcal{I}$ after $\alpha) \subseteq \operatorname{out}(\mathcal{S}$ after $\alpha)$

Fig. 3 shows a graphical representation of a specification and two tests generated from it. The specification models a coffee machine in which a user can press a button and either coffee or tea is returned. The specification has input actions ?Button1 and ?Button2 and output actions !Coffee and !Tea. A test derived from this specification is to do a ?Button input action and give a verdict pass if an output action!Coffee is observed from the implementation. If an output action !Tea is observed instead, then the test gives a verdict fail. If no output action is observed, indicated by the action $\theta$, then the test leads to a verdict fail as well. Another test is to do input action ?Button1 and if output action!Coffee is observed, do input action ?Button 2. In this case, if output action!Tea is observed a verdict pass is given and if output action!Coffee is observed a verdict fail is given.

In the ioco theory a test is a transition system with a tree-like structure and pass or fail verdicts as leaves. For tests an action $\theta$ is used instead of the action $\delta$ to indicate quiescence. This is done to make a difference between specified quiescence and observed quiescence. A test $\mathcal{T C}=\left(T \cup\{\right.$ pass, fail $\}, t_{0}, A_{I} \cup A_{O} \cup$ $\{\theta\}, \rightarrow \mathcal{T C})$ is a LTS with the following properties.

$-\mathcal{T C}$ is deterministic and has finite behavior.

- $\mathcal{T C}$ has two terminal states pass and fail. A terminal state of $\mathcal{T C}$ has no outgoing transitions. That is, there do not exist a $\mu \in A_{I} \cup A_{O} \cup\{\theta\}$ and a $t \in T$ such that pass $\stackrel{\mu}{\rightarrow}_{\mathcal{T C}} t$ or fail $\stackrel{\mu}{\rightarrow}_{\mathcal{T C}} t$. 


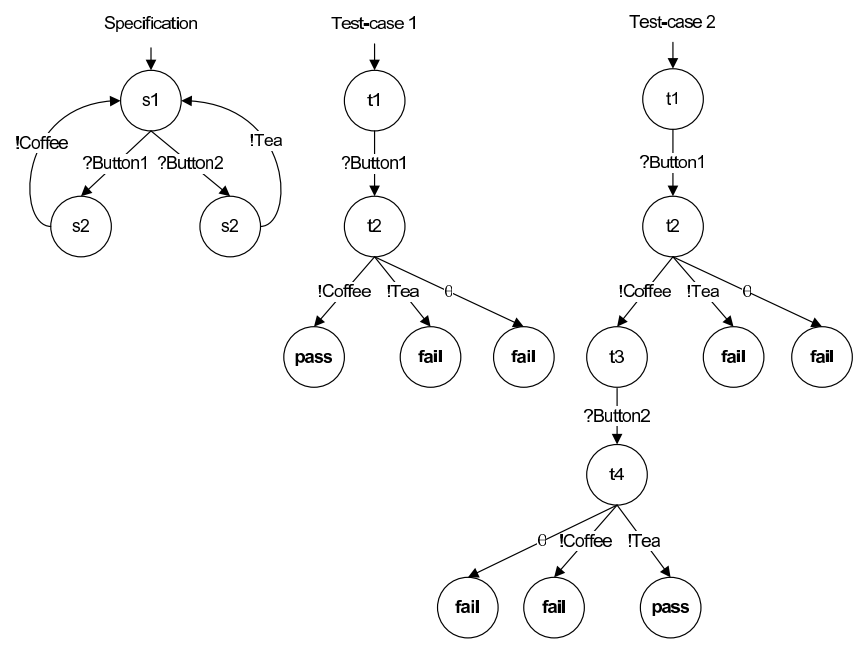

Fig. 3. Example Test Cases

A test is described using a process algebra like notation with an action prefix operator and an alternative composition operator for transition systems. The action prefix operator combines a LTS and an action $a$ to a new transition system. The alternative composition combines a set of LTSs into one LTS.

Definition 6. Let $\mathcal{L}=\left(S, s_{0}, A, \rightarrow\right)$ be a LTS. Let $s \notin S$ be a fresh state. The result of prefixing $\mathcal{L} \mathcal{T}$ with an action a, described by $a ; \mathcal{L}$, is defined by

$$
a ; \mathcal{L}=\left(S \cup\{s\}, s, A, \rightarrow \cup\left\{\left(t, a, t_{0}\right)\right\}\right) .
$$

Let $\mathcal{L} \mathcal{S}=\left\{\mathcal{L}_{i} \mid i \in I\right\}$ be a (possibly infinite) set of tests of the form $\mathcal{T C}_{i}=$ $\left(S_{i}, s_{0 i}, A_{i}, \rightarrow_{i}\right)$. Let $s \notin \bigcup_{i \in I} S_{i}$ be a fresh state. The alternative composition of $\mathcal{T C S}$, described by $\sum \mathcal{L S}$, is defined by

$$
\sum \mathcal{L S}=\left(\bigcup_{i \in I} S_{i} \cup\{s\}, s, \bigcup_{i \in I} A_{i}, \bigcup_{i \in I}\left(\rightarrow_{i} \cup\left\{\left(s, a, s^{\prime}\right) \mid \exists_{s^{\prime} \in S_{i}, a \in A_{i}}: s_{0 i} \stackrel{a}{\rightarrow} \mathcal{T} \mathcal{C}_{i} s^{\prime}\right\}\right)\right) .
$$

Furthermore, $\mathcal{L}_{0}+\mathcal{L}_{1}$ denotes $\sum\left\{\mathcal{L}_{0}, \mathcal{L}_{1}\right\}$, the LTS $(\{$ pass $\}$, pass, $\emptyset, \emptyset)$ is denoted by pass, and the LTS (\{fail $\}$, fail $, \emptyset, \emptyset)$ is denoted by fail.

A test describes which input actions are applied to the implementation and which output actions can be observed from the implementation. An output action leads to a verdict fail if the output action was not allowed according to the specification. If no output is observed, while according to the specification it should be observed, then observing quiescence $\theta$ leads to verdict fail. If according to the specification no output is possible then only the observation of $\theta$ is correct behavior of the implementation. In this case, an output action always leads to a verdict fail. The verdict pass is a test in itself. 
Definition 7. Let $\mathcal{S}=\left(S, s_{0}, A_{I} \cup A_{O}, \rightarrow\right)$ be a specification and let $C \subseteq S$ be a non-empty set of states; then the set of tests $\operatorname{Tests}_{\mathcal{S}}(C)$ is inductively defined as follows:

1. pass is an element of Tests $(C)$;

2. if $i \in A_{I}$ and $C$ after $i \neq \emptyset$ and $\mathcal{T} \mathcal{C}^{\prime} \in \operatorname{Tests}_{\mathcal{S}}(C$ after $i)$, then $i ; \mathcal{T} \mathcal{C}^{\prime}$ is an element of $\operatorname{Tests}_{\mathcal{S}}(C)$;

3. (a) if out $(C) \neq \emptyset$ and, for all $o \in \operatorname{out}(C), \mathcal{T C}_{o} \in \operatorname{Tests}_{\mathcal{S}}(C$ after $o$ ), then

$$
\sum\left\{o ; \mathcal{T C}_{o} \mid o \in A_{O} \cap \operatorname{out}(C)\right\}+\sum\left\{\mu ; \text { fail } \mid \mu \in\left(A_{O} \backslash \operatorname{out}(C)\right) \cup\{\theta\}\right\}
$$

is an element of $\operatorname{Tests}_{\mathcal{S}}(C)$; and

(b) If $\operatorname{out}(C)=\{\delta\}$ and $\mathcal{T C}_{\theta} \in$ Tests $_{\mathcal{S}}(C$ after $\theta)$, then

$$
\theta ; \mathcal{T C}_{\theta}+\sum\left\{o ; \text { fail } \mid o \in A_{O}\right\}
$$

is an element of $\operatorname{Tests}_{\mathcal{S}}(C)$.

The execution of a test on an implementation is formalized through the synchronous composition of the test with the implementation.

Definition 8. Let $\mathcal{T C}=\left(T, t_{0}, A_{I} \cup A_{O} \cup\{\delta\}, \rightarrow \mathcal{T C}\right)$ be a test and $\mathcal{I}=\left(S, s_{0}, A_{I} \cup\right.$ $\left.A_{O}, \rightarrow_{\mathcal{I}}\right)$ be an implementation, then the synchronous composition $\mathcal{T C} \| \mathcal{I}$ is defined by the LTS $\mathcal{T C} \| \mathcal{I}=\left(T \times S_{\mathcal{I}},\left(t_{0}, s_{0}\right), A_{I} \cup A_{O} \cup\{\delta\}, \rightarrow\right)$ with $\rightarrow=$ $\left\{\left((t, s), a,\left(t^{\prime}, s^{\prime}\right)\right) \mid \exists_{t, t^{\prime} \in T, s, s^{\prime} \in S_{\mathcal{I}}}: t \stackrel{a}{\rightarrow} \mathcal{T C} t^{\prime} \wedge s \stackrel{a}{\rightarrow} \mathcal{I} s^{\prime} \wedge a \in A_{I} \cup A_{O} \cup\{\delta\}\right\}$.

An implementation passes a test $\mathcal{T C}$ if all traces of the execution lead to a verdict pass. An implementation passes a set of tests $\mathcal{T C S}$ if all executions of tests in it lead to verdict pass. An implementation is input-output conform a specification if the execution all tests that can be generated from it lead to a verdict pass.

Definition 9. If $\mathcal{T C}=\left(T, t_{0}, \Sigma_{I} \cup \Sigma_{O} \cup\{\delta\}, \rightarrow_{t}\right)$ is a test and $\mathcal{I}=\left(S, s_{0}, \Sigma_{I} \cup\right.$ $\left.\Sigma_{O} \cup\{\delta\}, \rightarrow_{s}\right)$ is an IOTS, then $\mathcal{I}$ passes $\mathcal{T C}$ is defined as

$$
\mathcal{I} \text { passes } \mathcal{T C} \Longleftrightarrow \forall_{\alpha \in \operatorname{traces}(\mathcal{T C} \| \mathcal{I})}: \exists_{s^{\prime} \in S}:\left(t_{0}, s_{0}\right) \stackrel{\alpha}{\Rightarrow}\left(\text { pass, } s^{\prime}\right) .
$$

If $\mathcal{T C S}$ is a set of tests, then

$$
\mathcal{I} \text { passes } \mathcal{T C S} \Longleftrightarrow \forall_{\mathcal{T C} \in \mathcal{T} \mathcal{C S}}: \mathcal{I} \text { passes } \mathcal{T C} \text {. }
$$

The test generation procedure is sound in the sense that a correct implementation passes all tests generated from the specification. The procedure is exhaustive in the sense that for an incorrect implementation always a test can be generated that from the specification that leads to a verdict fail. 


\section{Hybrid Transition Systems}

Like in the discrete case, we define our conformance relation on the class of systems that can be described by a transition system. For hybrid systems we use hybrid transition systems [3]. In this report only introduce the definitions necessary for our theory. For instance, we use symbolic states and we leave open the initial valuation of variables (which can be defined by the initial state).

Before we can formally define hybrid transition systems, we first need to define trajectories. In order to define trajectories we need some mathematical preliminaries.

1. An interval over $\mathbb{R}$ is called left-closed if it has a minimum, right-closed if it has a maximum, and closed if it has both a minimum and a maximum.

2. Let $f: A \rightarrow B$ be a function; for $A^{\prime} \subseteq A$ we define $f$ restricted to $A^{\prime}$, denoted by $f\left\lceil A^{\prime}\right.$, as the function $f\left\lceil A^{\prime}: A^{\prime} \rightarrow B\right.$ defined by $f\left\lceil A^{\prime}(a)=f(a)\right.$ for $a \in A^{\prime}$.

3. Let $f: A \rightarrow B$ with $A \subseteq \mathbb{R}$ be a function and let $A+t=\{a+t \mid a \in A\}$; for $t \in \mathbb{R}$ we define $f+t: A+t \rightarrow B$ by $(f+t)\left(t^{\prime}\right)=f\left(t^{\prime}-t\right)$, with $t^{\prime} \in A+t$.

A trajectory is defined as a function from a interval to a vector of valuations of variables. A trajectory is defined over a right-closed interval of $\mathbb{R}$, starting at 0 .

\section{Definition 10.}

1. Let $V$ be a set of (continuous) variables. A valuation for $V$, denoted by $\operatorname{val}(V)$, is a function that associates with each variable $v \in V$ a value of the type of $v$. We write $\operatorname{val}(V)$ for the set of all valuations for $V$.

2. Let $t \in \mathbb{R}^{>0}$ be a positive real number and let $V$ be a set of variables. $A$ trajectory $\sigma$ is a function $\sigma:(0, t] \rightarrow \operatorname{val}(V)$ that associates with each element in the domain $(0, t]$ a valuation. We write trajs $(V)$ for the set of all trajectories with respect to $V$.

3. Let $\sigma$ be a trajectory. We write $\operatorname{dom}(\sigma)$ for the domain of $\sigma$, $\sigma$.fval for the first valuation of $\sigma$, $\sigma . l v a l$ for the last valuation of $\sigma$, and $\sigma . l$ time for the maximum of the domain of $\sigma$. Note that $\sigma(\sigma . l t i m e)=\sigma . l v a l$.

4. Let $\sigma$ and $\sigma^{\prime}$ be trajectories. The concatenation of $\sigma$ and $\sigma^{\prime}$ (denoted by $\left.\sigma \frown \sigma^{\prime}\right)$ is defined as:

$$
\sigma \frown \sigma^{\prime}=\sigma \cup\left(\sigma^{\prime}+\sigma . l \text { time }\right) .
$$

5. Let $\sigma$ be a trajectory on variables $V$; for $V^{\prime} \subseteq V$ we define $\sigma$ restricted to $V^{\prime}$, denoted by $\sigma \downarrow V^{\prime}$ as the function $\sigma \downarrow V^{\prime}: J \rightarrow\left(V^{\prime} \rightarrow \operatorname{val}\left(V^{\prime}\right)\right)$ defined by $\sigma \downarrow V^{\prime}(t)=\sigma(t)\left\lceil V^{\prime}\right.$ with $t \in J$.

6. Let $\sigma$ be a trajectory defined over an interval $(0, t]$, with $t>0$ and let $t^{\prime} \in \mathbb{R}^{>0}$ with $t^{\prime} \leq t$; then:

$$
\begin{aligned}
& \sigma \unlhd t^{\prime}=\sigma\left\lceil\left(0, t^{\prime}\right] ;\right. \\
& \sigma \unrhd t^{\prime}=\left(\sigma\left\lceil\left(t^{\prime}, t\right]\right)-t^{\prime} .\right.
\end{aligned}
$$


7. Let $\sigma$ and $\sigma^{\prime}$ be two trajectories; then $\sigma$ is a prefix of $\sigma^{\prime}$, denoted by $\sigma \leq \sigma^{\prime}$, if there exists a $t \in \mathbb{R}^{>0}$ such that $\sigma=\sigma^{\prime} \unlhd t$. We write $\sigma<\sigma^{\prime}$ if $\sigma \leq \sigma^{\prime}$ and $\sigma$.ltime $<\sigma^{\prime}$.ltime.

A hybrid transition system is a tuple consisting of a set of states, an initial state, a set of discrete transitions, and a set of continuous transitions. Every transition has a label. A label is either an action or a trajectory. The set of actions $A$ is partitioned into disjunct sets of input actions $A_{I}$ and output actions $A_{O}$ and internal action $\tau$, i.e. $A=A_{I} \uplus A_{O} \uplus\{\tau\}$. Every trajectory is defined on a set of variables $V$. $V$ is partitioned into disjunct sets of input variables $V_{I}$ and output variables $V_{O}$, i.e. $V=V_{I} \uplus V_{O}$.

A trajectory also models time. The interval over which the trajectory is defined is the time in which the flow takes place.

Definition 11. A hybrid transition system (HTS) is a tuple $\mathcal{H}=\left(S, s_{0}, \rightarrow, \rightsquigarrow\right)$, where

$-S$ is a (possibly infinite) set of states;

$-s_{0} \in S$ is the initial state;

$-\rightarrow \subseteq S \times A \times S$ is the set of discrete transitions for a set of actions A; and

- $\subseteq S \times \Sigma \times S$ is the set of continuous transitions for a set of trajectories $\Sigma$.

From now on, $\mathcal{H}$ always is a HTS $\mathcal{H}=\left(S, s_{0}, \rightarrow, \rightsquigarrow\right)$. We write $s \stackrel{a}{\rightarrow} s^{\prime}$ instead of $\left(s, a, s^{\prime}\right) \in \rightarrow$. We write $s \stackrel{\sigma}{\rightsquigarrow} s^{\prime}$ instead of $\left(s, \sigma, s^{\prime}\right) \in \rightsquigarrow$. We also write $s \stackrel{a}{\rightarrow}$ instead of $\exists_{s^{\prime} \in S}: s \stackrel{a}{\rightarrow} s^{\prime}$. and we write $s \stackrel{\sigma}{\rightsquigarrow}$ instead of $\exists_{s^{\prime} \in S}: s \stackrel{\sigma}{\rightsquigarrow} s^{\prime}$.

For our theory we need to assume three conditions on every HTS $\mathcal{H}$. The first condition we call trajectory interpolation, the second condition we call trajectory additivity, and the third condition we call trajectory determinism.

- A1: If $s \stackrel{\sigma^{\prime} \sim \sigma^{\prime \prime}}{\sim} s^{\prime \prime}$, then there exists a $s^{\prime} \in S$ such that $s \stackrel{\sigma^{\prime}}{\rightsquigarrow} s^{\prime}$ and $s^{\prime} \stackrel{\sigma^{\prime \prime}}{\rightsquigarrow} s^{\prime \prime}$.

- A2: If $s \stackrel{\sigma^{\prime}}{\rightsquigarrow} s^{\prime}$ and $s^{\prime} \stackrel{\sigma^{\prime \prime}}{\rightsquigarrow} s^{\prime \prime}$ then $s \stackrel{\sigma^{\prime} \sim \sigma^{\prime \prime}}{\sim} s^{\prime \prime}$.

- A3: If $s, s^{\prime}, s^{\prime \prime} \in S$ such that $s \stackrel{\sigma}{\sim} s^{\prime}$ and $s \stackrel{\sigma}{\sim} s^{\prime \prime}$ then $s^{\prime}=s^{\prime \prime}$.

These conditions are needed for a correct definition of tests. Note that we do not find it necessary to require that our systems are non Zeno. That is, we do allow traces with an infinite sequence of actions and trajectories that sum up to a finite amount of time. The reason we allow this behavior is that our conformance relation works on Zeno systems as well. However, in practice we only consider non Zeno systems because in practice time cannot stop.

The formal definition of input action enabling is that a HTS accepts any input action in every state. The definition of input trajectory enabling is that a HTS accepts any input trajectory in every state except in states in which only output actions or $\tau$ occur. All input trajectories are accepted either for the entire duration of that trajectory, or until the HTS interrupts the trajectory with an output action or $\tau$. After an interrupt the trajectory can still be completed because, since the system accepts any input trajectory, it accepts the suffix of the interrupted trajectory. 
Definition 12. Let $\mathcal{H}$ be a HTS.

- $\mathcal{H}$ is input action enabled if for every $s \in S$ and $i \in A_{I}: s \stackrel{i}{\rightarrow}$.

$-\mathcal{H}$ is input trajectory enabled if for every $s \in S:$

1. there exists an action $a \in A_{O} \cup\{\tau\}$ such that $s \stackrel{a}{\rightarrow}$ and there does not exist $a \sigma \in \Sigma$ such that $s \stackrel{\sigma}{\rightsquigarrow} ;$ or

2. for every $u \in \operatorname{trajs}\left(V_{I}\right)$ there exists $a \sigma \in \Sigma$ with $\sigma \downarrow V_{I} \leq u$ and:

(a) $\sigma \downarrow V_{I}=u$ and there exists an $s^{\prime} \in S: s \stackrel{\sigma}{\sim} s^{\prime}$; or

(b) $\sigma \downarrow V_{I}<u$ and there exists an action $a \in A_{O} \cup\{\tau\}$ and $s^{\prime} \in S$ such that: $s \stackrel{\sigma}{\rightsquigarrow} s^{\prime} \stackrel{a}{\rightarrow}$.

$-\mathcal{H}$ is input enabled if it is both input action enabled and input trajectory enabled.

The execution of a hybrid transition system is described by a sequence of observable actions and trajectories. A transition with an action or a trajectory leads to a state from which another transition can be taken. A sequence of which the start state is the initial state of the HTS is called a trace. Note that in our definition the internal action $\tau$ does not occur in a sequence or a trace.

Definition 13. Let $\mathcal{H}$ be a HTS.

1. We inductively define the generalized transition relation $\Rightarrow \subseteq S \times(A \cup \Sigma)^{*} \times S$ as the least relation that satisfies for all $s, s^{\prime}, s^{\prime \prime} \in S$ :

$-s \stackrel{\epsilon}{\Rightarrow} s$;

- if $s \stackrel{\tau}{\rightarrow} s^{\prime}$, then $s \stackrel{\epsilon}{\Rightarrow} s^{\prime}$;

- if $s \stackrel{a}{\rightarrow} s^{\prime}$, then $s \stackrel{a}{\Rightarrow} s^{\prime}$

- if $s \stackrel{\sigma}{\rightsquigarrow} s^{\prime}$, then $s \stackrel{\sigma}{\Rightarrow} s^{\prime}$; and

- if $s \stackrel{\alpha}{\Rightarrow} s^{\prime}$ and $s^{\prime} \stackrel{\beta}{\Rightarrow} s^{\prime \prime}$, then $s \stackrel{\alpha \beta}{\Rightarrow} s^{\prime \prime}$.

2. If $s \in S$ is a state of $S$, then a trace of $H T S \mathcal{H}$ is a sequence $\alpha \in(A \cup \Sigma)^{*}$ such that $s_{0} \stackrel{\alpha}{\Rightarrow} s$. The set of all traces of $\mathcal{H}$ is denoted by traces $(\mathcal{H})$.

For a state $s$ and a sequence $\alpha$ we also write $s \stackrel{\alpha}{\Rightarrow}$ instead of $\exists_{s^{\prime} \in S}: s \stackrel{\alpha}{\Rightarrow} s^{\prime}$. We denote by $s$ after $\alpha$ the set of reachable states from $s$ after a trace $\alpha$.

Definition 14. Let $\mathcal{H}$ be a HTS, $s \in S$ and $\alpha \in(A \cup \Sigma)^{*}$; then:

$$
s \text { after } \alpha=\left\{s^{\prime} \mid s \stackrel{\alpha}{\Rightarrow} s^{\prime}\right\} .
$$

For a set of states $C \subseteq S$ we define:

$$
C \text { after } \alpha=\bigcup_{c \in C} c \text { after } \alpha .
$$

We sometimes write $\mathcal{H}$ after $\alpha$ instead of $s_{0}$ after $\alpha$. 


\section{The Hybrid Input-output Conformance Relation}

In this section we define the conformance relation for hybrid systems. This conformance relation tells us whether a hybrid implementation conforms to a hybrid specification. This is the case if, in every reachable state, the implementation only contains specified discrete and continuous output behavior. The implementation is not conform a specification if, in some reachable state, the implementation performs an output action or a trajectory that is not specified.

Unlike in the ioco relation, where the concept of input-output transition systems is introduced, both the implementation and the specification are defined as hybrid transition systems. Like in the discrete case, an implementation is input enabled and a specification does not need to be input enabled.
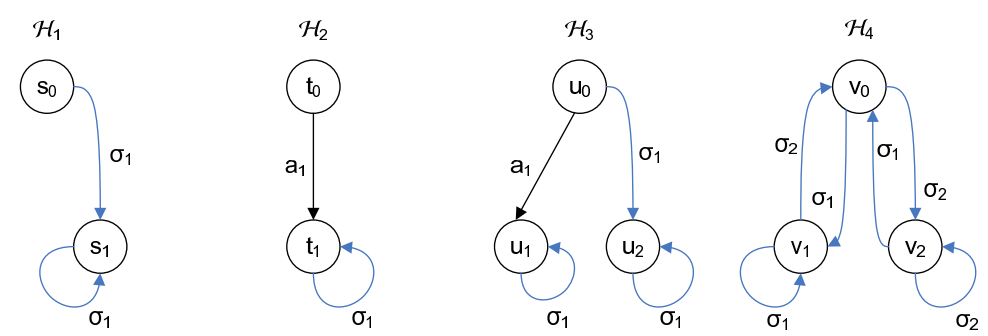

Fig. 4. Example Hybrid Transition Systems

Figure 4 shows four hybrid transition system examples. We cannot depict all transitions and states such that $\mathbf{A 1}$ to $\mathbf{A} \mathbf{3}$ hold, because there are infinitely many, but we assume all these transitions are included in the example systems. These examples are not input-enabled. Still, they can be used to illustrate our theory.

Intuitively, $\mathcal{H}_{3}$ is not conform $\mathcal{H}_{1}$ because $\mathcal{H}_{3}$ can perform an output action (in state $u_{0}$ ), while $\mathcal{H}_{1}$ cannot. On the other hand, $\mathcal{H}_{1}$ conforms to $\mathcal{H}_{3}$ because $\mathcal{H}_{3}$ allows all the behavior that $\mathcal{H}_{1}$ can display. $\mathcal{H}_{3}$ is not conform $\mathcal{H}_{2}$ because when according to $\mathcal{H}_{2}$ an output action has to happen, according to $\mathcal{H}_{3}$ it may not happen. On the other hand, $\mathcal{H}_{2}$ conforms to $\mathcal{H}_{3}$ because when the output action $a_{1}$ occurs, it can also occur according to $\mathcal{H}_{3}$. If the flow of input variables in trajectory $\sigma_{1}$ is different from the flow of input variables in trajectory $\sigma_{2}$ (i.e., $\left.\sigma_{1} \downarrow V_{I} \neq \sigma_{2} \downarrow V_{I}\right)$, then $\mathcal{H}_{4}$ conforms to $\mathcal{H}_{1}$. The reason is that with respect to the input trajectory $\mathcal{H}_{4}$ displays the same output behavior as $\mathcal{H}_{1}$. However, if the flow of input variables is the same for $\sigma_{1}$ and $\sigma_{2}$ (or there are no input variables) and the flow of output variables is not the same for $\sigma_{1}$ and $\sigma_{2}$, then $\mathcal{H}_{4}$ is not conform $\mathcal{H}_{1}$ because $\mathcal{H}_{4}$ can display output behavior that is not allowed according to $\mathcal{H}_{1}$.

For our conformance relation we first define the set of trajectories allowed in a state $s$ by $\operatorname{traj}(s)$ and we define the trajectories allowed in a set of states $C$ 
by $\operatorname{traj}(C)$. We need to define the conformance relation on both input trajectories and output trajectories because of the dependency between them in hybrid systems.

Definition 15. Let $\mathcal{H}$ be a HTS and let $s \in S$ be a state of $\mathcal{H}$; then:

$$
\operatorname{traj}(s)=\{\sigma \in \Sigma \mid s \stackrel{\sigma}{\rightsquigarrow}\} .
$$

For a set of states $C \subseteq S$ we define:

$$
\operatorname{traj}(C)=\bigcup_{c \in C} \operatorname{traj}(c) .
$$

For instance, $\operatorname{traj}\left(s_{0}\right)$ of $\mathcal{H}_{1}$ is $\left\{\sigma_{1}\right\}$ and $\operatorname{traj}\left(u_{0}\right)$ of $\mathcal{H}_{4}$ is $\left\{\sigma_{1}, \sigma_{2}\right\}$.

Since an implementation is input enabled and a specification does not have to be input enabled, the specification determines which trajectories are relevant in our relation. Namely, those trajectories for which an input trajectory exists in the specification. In case we determine whether $\mathcal{H}_{4}$ conforms to $\mathcal{H}_{3}$ : if $\sigma_{1} \downarrow V_{I} \neq$ $\sigma_{2} \downarrow V_{I}$, then we only want to take into account $\sigma_{1}$; and if $\sigma_{1} \downarrow V_{I}=\sigma_{2} \downarrow V_{I}$, then we want to take into account $\sigma_{1}$ and $\sigma_{2}$.

Definition 16. Let $\Sigma_{\mathcal{I}}$ and $\Sigma_{\mathcal{S}}$ be two sets of trajectories on a set of variables $V$ with input variables $V_{I} \subseteq V$; then:

$$
\operatorname{infilter}\left(\Sigma_{\mathcal{I}}, \Sigma_{\mathcal{S}}\right)=\left\{\sigma \in \Sigma_{\mathcal{I}} \exists_{\sigma^{\prime} \in \Sigma_{\mathcal{S}}}: \sigma \downarrow V_{I}=\sigma^{\prime} \downarrow V_{I}\right\} .
$$

For instance, if $\sigma_{1} \downarrow V_{I} \neq \sigma_{2} \downarrow V_{I}$, then $\operatorname{infilter}\left(\operatorname{traj}\left(v_{0}\right), \operatorname{traj}\left(s_{0}\right)\right)=\left\{\sigma_{1}\right\}$.

As described earlier, in a hybrid transition system time progresses through trajectories. Actions are instantaneous. It is possible to specify that time cannot progress unless an action happens first. It allows us to specify urgent actions, by states from which exactly one action is possible and no trajectories are possible. Unfortunately, with this infilter, we might loose the information whether according to implementation an (output) action had to happen or could be delayed. For instance, if we apply the infilter with $\mathcal{H}_{3}$ as implementation and $\mathcal{H}_{2}$ as specification; then, in the initial states $u_{0}$ and $t_{0}$ respectively, the resulting set of trajectories turns out to be empty $\left(\operatorname{infilter}\left(\operatorname{traj}\left(u_{0}\right), \operatorname{traj}\left(s_{0}\right)\right)=\emptyset\right)$. The information that $a_{1}$ did not have to happen is lost. To solve this problem we use a symbol $\xi$ which indicates that, in a state, besides output actions also trajectories are allowed.

Definition 17. Let $\mathcal{H}$ be a HTS and let $s \in S$ be a state of $\mathcal{H}$; then:

$$
\operatorname{out}(s)= \begin{cases}\left\{o \in A_{O} \mid \exists_{s^{\prime} \in S}: s \stackrel{o}{\rightarrow} s^{\prime}\right\} \cup\{\xi\}, \text { if } \exists_{\sigma \in \Sigma, s^{\prime} \in S}:\left(s \stackrel{\sigma}{\rightsquigarrow} s^{\prime}\right) ; \\ \left\{o \in A_{O} \mid \exists_{s^{\prime} \in S}: s \stackrel{o}{\rightarrow} s^{\prime}\right\} \quad, \text { otherwise. }\end{cases}
$$

For a set of states $C \subseteq S$ we define:

$$
\operatorname{out}(C)=\bigcup_{c \in C} \operatorname{out}(c)
$$


For instance, $\operatorname{out}\left(s_{0}\right)$ of $\mathcal{H}_{1}$ is $\{\xi\}$ and $\operatorname{out}\left(u_{0}\right)$ of $\mathcal{H}_{3}$ is $\left\{a_{1}, \xi\right\}$.

Finally we can define our conformance relation for hybrid systems called hioco. Informally, in every reachable state the set of output actions and the set of trajectories performed by the implementation, filtered on input allowed by the specification, should be a subset of the set of output actions and the set of trajectories in the specification.

Definition 18. Let $\mathcal{S}$ be a HTS and let $\mathcal{I}$ be an input enabled HTS. We say that $\mathcal{I}$ is input-output conform $\mathcal{S}$ (notation: $\mathcal{I}$ hioco $\mathcal{S}$ ) iff:

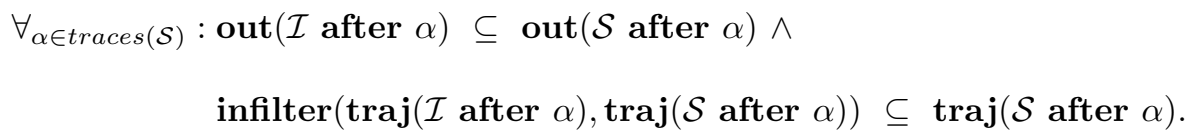

Checking the conformance using our relation for our example systems with respect to some of the others has the following results.

- $\mathcal{H}_{1}$ is input-output conform $\mathcal{H}_{3}$.

- $\mathcal{H}_{2}$ is input-output conform $\mathcal{H}_{3}$.

- $\mathcal{H}_{3}$ is not input-output conform $\mathcal{H}_{2}$ because after the trace $\epsilon$, out $\left(t_{0}\right)=$ $\left\{a_{1}, \xi\right\}$ and $\operatorname{out}\left(s_{0}\right)=\{\xi\}$ and therefore $\operatorname{out}\left(t_{0}\right) \not \subset \operatorname{out}\left(s_{0}\right)$.

- If we assume that $\sigma_{1} \neq \sigma_{2}$ and $\sigma_{1} \downarrow V_{I} \neq \sigma_{2} \downarrow V_{I}$, then $\mathcal{H}_{4}$ is input-output conform $\mathcal{H}_{1}$ and $\mathcal{H}_{4}$ is input-output conform $\mathcal{H}_{3}$.

- If we assume that $\sigma_{1} \neq \sigma_{2}$ and $\sigma_{1} \downarrow V_{I}=\sigma_{2} \downarrow V_{I}$, then $\mathcal{H}_{4}$ is not inputoutput conform $\mathcal{H}_{1}$ because infilter $\left(\left\{\sigma_{1}, \sigma_{2}\right\},\left\{\sigma_{1}\right\}\right)=\left\{\sigma_{1}, \sigma_{2}\right\}$ and therefore $\left\{\sigma_{1}, \sigma_{2}\right\} \nsubseteq \operatorname{traj}\left(t_{0}\right)$.

These results comply with our intuition about when these examples should be conform each other or not.

\section{Tests for Hybrid Systems}

In the rest of this report, HTS $\mathcal{S}=\left(S_{\mathcal{S}}, s_{0 \mathcal{S}}, \rightarrow \mathcal{S}, \rightsquigarrow \mathcal{S}\right)$ always is a specification and HTS $\mathcal{I}=\left(S_{\mathcal{I}}, s_{0 \mathcal{I}}, \rightarrow_{\mathcal{I}}, \rightsquigarrow \mathcal{I}\right)$ always is an implementation. For testing the conformance between a hybrid implementation and a hybrid specification we associate a set of tests with the specification. A hybrid test is a hybrid transition system $\mathcal{T C}=\left(T \cup\{\right.$ pass, fail $\left.\}, t_{0}, \rightarrow_{\mathcal{T C}}, \rightsquigarrow_{\mathcal{T C}}\right)$. Like in case of test generation for discrete systems a test has a tree like structure and two terminal states pass or fail as leaves. Besides being deterministic for actions, a hybrid test is also deterministic with respect to trajectories. A hybrid test has the following properties.

- The states pass and fail are terminal states of the test. That is, there does not exist $a \in A \cup \Sigma_{\mathcal{T C}}$ such that pass $\stackrel{a}{\rightarrow}$ or fail $\stackrel{a}{\rightarrow}$.

- A test is deterministic with respect to actions. That is, for all $t, t^{\prime}, t^{\prime \prime} \in T$ and $a \in A$, if $t \stackrel{a}{\rightarrow} \mathcal{T C} t^{\prime}$ and $t \stackrel{a}{\rightarrow} \mathcal{T C} t^{\prime \prime}$, then $t^{\prime}=t^{\prime \prime}$. 
- A test is strongly time deterministic with respect to trajectories.

- The conditions A1, A2, and A3 hold for tests as well.

Tests are described by a process algebra like notation for action prefixing or trajectory prefixing, and alternative composition. I.e., $a ; \mathcal{T C}$ denotes a test prefixed by an action $a, \sigma ; \mathcal{T C}$ denotes a test prefixed by a trajectory $\sigma, \sum \mathcal{T} \mathcal{S}$ denotes the alternative composition of a set of tests $\mathcal{T S}$. We write $\mathcal{T C}+\mathcal{T} \mathcal{C}^{\prime}$ instead of $\sum\left\{\mathcal{T C}, \mathcal{T} \mathcal{C}^{\prime}\right\}$.

\section{Definition 19.}

1. Let $a$ be an action, and let $\mathcal{H}$ be a HTS. Then a; $\mathcal{H}$, the HTS that results from prefixing $\mathcal{H}$ with the action $a$, is defined by

$$
a ; \mathcal{H}=\left(S \cup\{s\}, t, \rightarrow \cup\left\{\left(s, a, s_{0}\right)\right\}, \rightsquigarrow\right) .
$$

(It is assumed in the definition above that $s \notin S$.)

2. Let $\sigma$ be a trajectory, and let $\mathcal{H}$ be a HTS. Then $\sigma ; \mathcal{H}$, the HTS that results from prefixing $\mathcal{H}$ with the trajectory $\sigma$, is defined by

$$
\sigma ; \mathcal{H}=\left(S \cup S^{\prime}, s_{r}, \rightarrow, \rightsquigarrow \cup \rightsquigarrow^{\prime}\right),
$$

where

$$
S^{\prime}=\left\{s_{r^{\prime}} \mid r \geq r^{\prime}>0\right\}
$$

and

$$
\begin{aligned}
& \rightsquigarrow^{\prime}=\left\{\left(s_{r^{\prime}},\left(\sigma \unrhd\left(r-r^{\prime}\right)\right) \unlhd\left(r^{\prime}-r^{\prime \prime}\right), s_{r^{\prime \prime}}\right) \mid r \geq r^{\prime}>r^{\prime \prime} \geq 0\right\} \\
& \cup\left\{\left(s_{r^{\prime}},\left(\sigma \unrhd\left(r-r^{\prime}\right)\right) \frown \sigma^{\prime}, s^{\prime}\right) \mid r \geq r^{\prime}>0 \wedge s_{0} \stackrel{\sigma^{\prime}}{\rightsquigarrow} s^{\prime}\right\} .
\end{aligned}
$$

(It is assumed in the definition above that $S \cap S^{\prime}=\emptyset$ and that $s_{r^{\prime}}=s_{r^{\prime \prime}}$ implies $r^{\prime}=r^{\prime \prime}$ for all $r \geq r^{\prime}, r^{\prime \prime}>0$.)

3. Let $\mathcal{H S}=\left\{\mathcal{H}_{i} \mid i \in I\right\}$ with $\mathcal{H}_{i}=\left(S_{i}, s_{0 i}, \rightarrow_{i}, \rightsquigarrow_{i}\right)$ be a set of hybrid transition systems. We define the summation $\sum \mathcal{H S}$ by

$$
\sum \mathcal{H S}=\left(\bigcup_{i \in I} S_{i} \cup S^{\prime} \cup\left\{s_{0}\right\}, s_{0}, \bigcup_{i \in I} \rightarrow_{i} \cup \rightarrow^{\prime}, \bigcup_{i \in I} \rightsquigarrow_{i} \cup \rightsquigarrow^{\prime}\right),
$$

where

$$
\begin{gathered}
S^{\prime}=\left\{s_{\sigma} \mid \sigma \text { a trajectory s.t. } s_{0 i} \stackrel{\sigma}{\rightsquigarrow} \text { for some } i \in I\right\}, \\
\rightarrow^{\prime}=\left\{\left(s_{0}, a, s^{\prime}\right) \mid \exists i \in I . s_{0 i} \stackrel{a}{\rightarrow}_{i} s^{\prime}\right\} \\
\cup\left\{\left(s_{\sigma}, a, s^{\prime}\right) \mid \exists i \in I, s^{\prime \prime} . s_{0 i} \stackrel{\sigma}{\hookrightarrow}_{i} s^{\prime \prime} \stackrel{a}{\rightarrow}_{i} s^{\prime}\right\},
\end{gathered}
$$

and

$$
\rightsquigarrow^{\prime}=\left\{\left(s_{0}, \sigma, s_{\sigma}\right) \mid s_{\sigma} \in S^{\prime}\right\} \cup\left\{\left(s_{\sigma}, \sigma^{\prime \prime}, s_{\sigma^{\prime}}\right) \mid s_{\sigma}, s_{\sigma^{\prime}} \in S^{\prime} \wedge \sigma \frown \sigma^{\prime \prime}=\sigma^{\prime}\right\} .
$$

(It is assumed in the definition above that $\left(\bigcup_{i \in I} S_{i}\right) \cap\left(S^{\prime} \cup s_{0}\right)=\emptyset$.) 
We also write pass for the HTS (\{pass $\}$, pass, $\emptyset, \emptyset)$ and we write fail for the $\operatorname{HTS}(\{$ fail $\}$, fail $, \emptyset, \emptyset)$.

A test is associated to a specification as follows. If according to the specification some input actions are allowed, the test can allow one of these input actions. If according to the specification some output actions are allowed but no trajectories, the allowed output actions may lead to the verdict pass or continuation of the test. The other output actions and all trajectories lead to the verdict fail. If according to the specification trajectories are allowed, a particular input trajectory is chosen. If the complete trajectory (including output variables) is allowed according to the specification, then the test may lead to the verdict pass or testing may be continued. All other trajectories lead to the verdict fail. It may be that applying the selected input trajectory and observing the output trajectory is interrupted by an output action. If this interruption is allowed according to the specification, then the test may be continued or the verdict pass may be given. If the output action was not allowed the verdict fail is given.
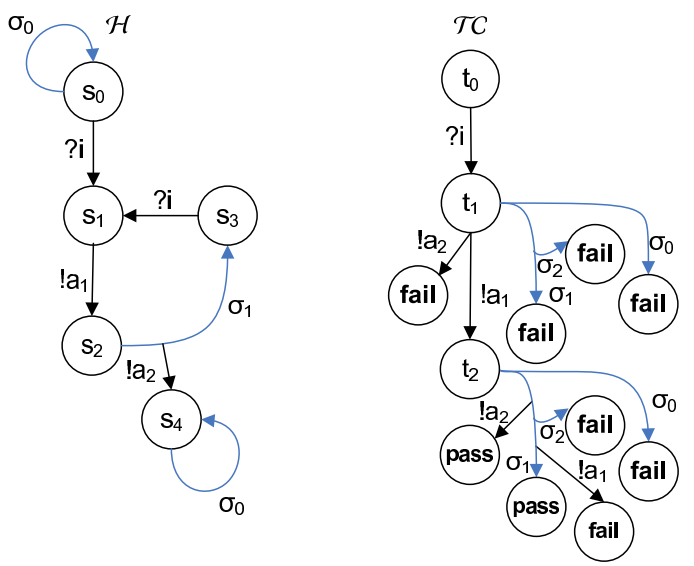

Fig. 5. Example Hybrid Test

Figure 5 depicts an illustrative (but not complete) test $\mathcal{T C}$ generated from a hybrid system $\mathcal{H}$. In our displayed system the transition with output action $! a_{2}$ means that trajectory $\sigma_{1}$ is interrupted. Note that also for this example we cannot depict all the transitions and states such that $\mathbf{A} \mathbf{1}$ to $\mathbf{A} \mathbf{3}$ hold.

The example test $\mathcal{T C}$ says first to apply the input action ? $i$ to the implementation, and immediately observe the output action $! a_{1}$. The observation of all other behavior (output actions or trajectories) leads to the verdict fail. After that, either the complete trajectory $\sigma_{1}$ or the prefix of this trajectory followed by output action ! $a_{2}$ is correct behavior and leads to a verdict pass. All other behavior leads to the verdict fail. 
Definition 20. Let $\mathcal{S}$ be a specification and let $C \subseteq S$ be a non-empty set of states; then the set of tests Tests $\mathcal{S}_{\mathcal{S}}(C)$ is inductively defined as follows:

1. pass is an element of Tests $(C)$;

2. if $i \in A_{I}$ and $C$ after $i \neq \emptyset$ and $\mathcal{T} \mathcal{C}^{\prime} \in \operatorname{Tests}_{\mathcal{S}}(C$ after $i)$, then $i ; \mathcal{T} \mathcal{C}^{\prime}$ is an element of $\operatorname{Tests}_{\mathcal{S}}(C)$;

3. if $\operatorname{traj}(C)=\emptyset$ and, for all $o \in A_{O}$ with $o \in \operatorname{out}(C), \mathcal{T C}_{o} \in \operatorname{Tests}_{\mathcal{S}}(C$ after $o)$, then

$$
\begin{aligned}
& \sum\left\{o ; \mathcal{T} \mathcal{C}_{o} \mid o \in A_{O} \cap \operatorname{out}(C)\right\}+ \\
& \sum\left\{o ; \text { fail } \mid o \in A_{O} \backslash \text { out }(C)\right\}+ \\
& \sum\left\{\sigma ; \text { fail } \mid \sigma \in \Sigma_{\mathcal{S}}\right\}
\end{aligned}
$$

is an element of $\operatorname{Tests}_{\mathcal{S}}(C)$; or

4. if

- $u \in\left\{\sigma \downarrow V_{I} \mid \sigma \in \operatorname{traj}(C)\right\}$ is an input trajectory and

- $\operatorname{traj}_{\mathbf{u}}(C)=\left\{\sigma \mid \sigma \downarrow V_{I}=u \wedge \sigma \in \operatorname{traj}(C)\right\}$ is the set of trajectories with input trajectory $u$ and

- subtraju $\mathbf{j}_{\mathbf{u}}(C)=\left\{\sigma \mid \exists_{\sigma^{\prime} \in \operatorname{traj}_{\mathbf{u}}(C)}: \sigma \leq \sigma^{\prime}\right\}$ is the set of prefixes of the set of trajectories traju $(C)$,

and $j=$ u.ltime and for all $\sigma \in \operatorname{traj}_{\mathbf{u}}(C), \mathcal{T C}_{\sigma} \in$ Tests $_{\mathcal{S}}(C$ after $\sigma)$ and for all $\sigma^{\prime} \in \operatorname{subtraj}_{\mathbf{u}}(C)$ and $o \in \operatorname{out}\left(C\right.$ after $\left.\sigma^{\prime}\right), \mathcal{T C}_{\sigma^{\prime} o} \in T e s t s\left(C\right.$ after $\left.\sigma^{\prime} o\right)$, then

$$
\begin{aligned}
& \sum\left\{\sigma ; T C_{\sigma} \mid \sigma \in \operatorname{traj}_{\mathbf{u}}(C)\right\}+\sum\left\{\sigma ; \text { fail } \mid \sigma \notin \text { subtraju }_{\mathbf{u}}(C)\right\}+ \\
& \sum\left\{o ; \mathcal{T C}_{o} \mid o \in A_{O} \cap \text { out }(C)\right\}+\sum\left\{o ; \text { fail } \mid o \in A_{O} \backslash \text { out }(C)\right\}+ \\
& \sum\left\{\sigma^{\prime} ; o ; \mathcal{T C}_{\sigma^{\prime}} \mid \sigma^{\prime} \in \operatorname{subtraj}(C) \wedge \sigma^{\prime} . l t i m e<j \wedge o \in \text { out }\left(C \text { after } \sigma^{\prime}\right)\right\}+ \\
& \sum\left\{\sigma^{\prime} ; o ; \text { fail } \mid \sigma^{\prime} \in \text { subtraj }_{\mathbf{u}}(C) \wedge \sigma^{\prime} . l t i m e<j \wedge o \notin \text { out }\left(C \text { after } \sigma^{\prime}\right)\right\}
\end{aligned}
$$

is an element of $\operatorname{Tests}_{\mathcal{S}}(C)$.

Note that in a test we do not need a special action to observe the symbol $\xi$ that we used in our conformance relation because $\xi$ can be observed by applying and observing a trajectory.

The execution of a test is defined by the synchronous composition of the test and the implementation.

Definition 21. Let $\mathcal{T C}$ be a test and $\mathcal{I}$ be an implementation. The synchronous composition of $\mathcal{T C}$ is defined as $\mathcal{T C} \| \mathcal{I}=\left(S,\left(t_{0}, s_{0}\right), \rightarrow, \rightsquigarrow\right)$ with:

$$
\begin{aligned}
& -S=T \times S_{\mathcal{I}} \\
& -\rightarrow=\left\{(t, s) \stackrel{a}{\rightarrow}\left(t^{\prime}, s^{\prime}\right) \mid t \stackrel{a}{\rightarrow} \mathcal{T C} t^{\prime} \wedge s \stackrel{a}{\rightarrow}_{\mathcal{I}} s^{\prime} \wedge a \in A\right\} \cup\left\{(t, s) \stackrel{\tau}{\rightarrow}\left(t, s^{\prime}\right) \mid s \stackrel{\tau}{\rightarrow}_{\mathcal{I}} s^{\prime}\right\} ; \\
& -\rightsquigarrow=\left\{(t, s) \stackrel{\sigma}{\rightsquigarrow}\left(t^{\prime}, s^{\prime}\right) \mid t \stackrel{\sigma}{\rightsquigarrow} \mathcal{T C} t^{\prime} \wedge s \stackrel{\sigma}{\rightsquigarrow} \mathcal{I} s^{\prime} \wedge \sigma \in \Sigma\right\} .
\end{aligned}
$$

Definition 22. Let $\mathcal{T C}$ be a test and $\mathcal{I}$ be an implementation. The set of test runs, defined by testruns $(\mathcal{T C} \| \mathcal{I})$, is the set of all traces that lead to a state pass or fail.

$$
\operatorname{testruns}(\mathcal{T C} \| \mathcal{I})=\left\{\alpha \mid \exists_{s \in S_{\mathcal{I}}}:\left(t_{0}, s_{0}\right) \stackrel{\alpha}{\Rightarrow}(\text { pass }, s) \vee\left(t_{0}, s_{0}\right) \stackrel{\alpha}{\Rightarrow}(\text { fail }, s)\right\}
$$

We say a hybrid implementation passes a hybrid test if only the verdict pass is reachable in the execution of the test. 
Definition 23. If $\mathcal{T C}$ is a test and $\mathcal{I}$ is an implementation, then $\mathcal{I}$ passes $\mathcal{T C}$ is defined as

$\mathcal{I}$ passes $\mathcal{T C} \Longleftrightarrow \forall_{\alpha \in \text { testruns }(\mathcal{T} \mathcal{C} \| \mathcal{I})}: \exists_{s^{\prime} \in S}:\left(t_{0}, s_{0}\right) \stackrel{\alpha}{\Rightarrow}\left(\right.$ pass, $\left.s^{\prime}\right)$.

If $\mathcal{T C S}$ is a set of tests, then

$\mathcal{I}$ passes $\mathcal{T C S} \Longleftrightarrow \forall_{\mathcal{T C} \in \mathcal{T C S}}: \mathcal{I}$ passes $\mathcal{T C}$.

\section{Soundness and Exhaustiveness of Hybrid Tests}

In this section we prove the soundness and exhaustiveness of our tests. In order to prove these we need some extra definitions.

Definition 24. Let $\mathcal{T C} \in$ Tests $_{\mathcal{S}}(C)$ be a test and let $t$ be a state of $\mathcal{T C}$. Then, $\mathcal{T} \mathcal{C}^{\prime}=\left(T^{\prime} \cup\{\right.$ pass, fail $\left.\}, t, \rightarrow^{\prime}, \rightsquigarrow^{\prime}\right)$ is a subtest of $\mathcal{T C}$ if:

$-T^{\prime} \subseteq T, \rightarrow^{\prime} \subseteq \rightarrow, \rightsquigarrow^{\prime} \subseteq \rightsquigarrow$,

- every reachable state of $\mathcal{T} \mathcal{C}^{\prime}$ is also reachable in $\mathcal{T C}$, and

- there exists an $\alpha \in \operatorname{traces}(\mathcal{T C})$ such that $t_{0} \stackrel{\alpha}{\Rightarrow} t$ and $\mathcal{T C}^{\prime} \in \operatorname{Tests}_{\mathcal{S}}(C$ after $\alpha)$.

Definition 25. We inductively define the normalized trace ntrace $(\alpha)$ of trace $\alpha$ as:

- if $\alpha=\epsilon$ then ntrace $(\alpha)=\epsilon$

- if $\alpha=a$, for some action $a$, then $\operatorname{ntrace}(\alpha)=a$;

- if $\alpha=\sigma$ then $\operatorname{ntrace}(\alpha)=\sigma$;

- if $\alpha=a \alpha^{\prime}$ then $\operatorname{ntrace}(\alpha)=a \operatorname{ntrace}\left(\alpha^{\prime}\right)$;

- if $\alpha=\sigma \alpha^{\prime}$ and ntrace $\left(\alpha^{\prime}\right)=a \alpha^{\prime \prime}$ then $\operatorname{ntrace}(\alpha)=a \operatorname{ntrace}\left(\alpha^{\prime}\right)$; and

- if $\alpha=\sigma \alpha^{\prime}$ and $\operatorname{ntrace}\left(\alpha^{\prime}\right)=\sigma \alpha^{\prime \prime}$ then $\operatorname{ntrace}(\alpha)=\sigma \frown \sigma^{\prime}$ ntrace $\left(\alpha^{\prime \prime}\right)$.

Lemma 1. Let $\alpha$ be a trace and let $\mathcal{H}$ be a hybrid transition system. If $\alpha \in$ traces $(\mathcal{H})$ then $\operatorname{ntrace}(\alpha) \in \operatorname{traces}(\mathcal{H})$.

This lemma holds because of conditions $\mathbf{A} \mathbf{1}$ and $\mathbf{A 2}$ which we imposed on hybrid transition systems.

Let $\mathcal{T C}$ be a test and let $\mathcal{T} \mathcal{C}^{\prime}$ be a subtest of $\mathcal{T C}$. Let $t_{0}, t_{0}^{\prime} \in T$ such that $t_{0}$ is the initial state of $\mathcal{T C}$ and $t_{0}^{\prime}$ is the initial state of subtest $\mathcal{T} \mathcal{C}^{\prime}$. Then, we write $\mathcal{T C} \stackrel{\alpha}{\Rightarrow} \mathcal{T} \mathcal{C}^{\prime}$ instead of $t_{0} \stackrel{\alpha}{\Rightarrow} t_{0}^{\prime}$ and $\mathcal{T C} \stackrel{\alpha}{\Rightarrow}$ fail instead of $t_{0} \stackrel{\alpha}{\Rightarrow}$ fail. We also write $\mathcal{T C} \stackrel{a}{\rightarrow} \mathcal{T} \mathcal{C}^{\prime}$ instead of $t_{0} \stackrel{a}{\rightarrow} t_{0}^{\prime}, \mathcal{T C} \stackrel{\sigma}{\rightsquigarrow} \mathcal{T} \mathcal{C}^{\prime}$ instead of $t_{0} \stackrel{\sigma}{\rightsquigarrow} t_{0}^{\prime}, \mathcal{T C} \stackrel{a}{\rightarrow}$ fail instead of $t_{0} \stackrel{a}{\Rightarrow}$ fail, and $\mathcal{T C} \stackrel{\sigma}{\rightsquigarrow}$ fail instead of $t_{0} \stackrel{\sigma}{\Rightarrow}$ fail.

Lemma 2. If $\mathcal{T C} \in \operatorname{Tests}_{\mathcal{S}}(C)$ and $\alpha \in \operatorname{testruns}(\mathcal{T C} \| \mathcal{I})$ such that $\mathcal{T C} \stackrel{\alpha}{\Rightarrow}$ fail, then there exist a trace $\alpha^{\prime} \in \operatorname{traces}(C)$, a subtest $\mathcal{T} \mathcal{C}^{\prime}$, and an output action $o \in A_{O}$ or a trajectory $\sigma$ such that either: 
1. $\operatorname{ntrace}(\alpha)=\alpha^{\prime} o, \mathcal{T} \mathcal{C}^{\prime} \in \operatorname{Tests}_{\mathcal{S}}\left(C\right.$ after $\left.\alpha^{\prime}\right), \mathcal{T C} \stackrel{\alpha^{\prime}}{\Rightarrow} \mathcal{T} \mathcal{C}^{\prime}$, and $\mathcal{T C}^{\prime} \stackrel{o}{\rightarrow}$ fail;

2. ntrace $(\alpha)=\alpha^{\prime} \sigma, \mathcal{T} \mathcal{C}^{\prime} \in \operatorname{Tests}_{\mathcal{S}}\left(C\right.$ after $\left.\alpha^{\prime}\right), \mathcal{T C} \stackrel{\alpha^{\prime}}{\Rightarrow} \mathcal{T} \mathcal{C}^{\prime}$ and $\mathcal{T} \mathcal{C}^{\prime} \stackrel{\sigma}{\rightsquigarrow}$ fail; or

3. $\operatorname{ntrace}(\alpha)=\alpha^{\prime} \sigma o, \mathcal{T} \mathcal{C}^{\prime} \in \operatorname{Tests}_{\mathcal{S}}\left(C\right.$ after $\left.\alpha^{\prime}\right), \mathcal{T C} \stackrel{\alpha^{\prime}}{\Rightarrow} \mathcal{T} \mathcal{C}^{\prime}$ and $\mathcal{T} \mathcal{C}^{\prime} \stackrel{\sigma o}{\Rightarrow}$ fail.

Proof. We prove this lemma by induction on the structure of $\mathcal{T C} \in \operatorname{Tests}_{\mathcal{S}}(C)$ and $\alpha \in$ testruns $(\mathcal{T C} \| \mathcal{I})$ such that $\mathcal{T C} \stackrel{\alpha}{\Rightarrow}$ fail.

1. Suppose that $\mathcal{T C}=$ pass. Then, Lemma 2 vacuously holds, since $\mathcal{T C} \stackrel{\alpha}{\Rightarrow}$ fail is not possible.

2. Suppose that $\mathcal{T C}=i ; \mathcal{T C}^{\prime}$, with $i \in A_{I}$ and $\mathcal{T C}^{\prime} \in \operatorname{Tests}_{\mathcal{S}}(C$ after $i)$ and Lemma 2 holds for every $\alpha^{\prime} \in \operatorname{testruns}\left(\mathcal{T} \mathcal{C}^{\prime} \| \mathcal{I}\right)$ for which $\mathcal{T} \mathcal{C}^{\prime} \stackrel{\alpha^{\prime}}{\Rightarrow}$ fail (the induction hypothesis). Then, since $\mathcal{T C} \stackrel{i}{\rightarrow} \mathcal{T} \mathcal{C}^{\prime}$ and, by the induction hypothesis, Lemma 1 holds for $\mathcal{T C}^{\prime}$ and $\alpha^{\prime}$, it follows that Lemma 2 holds for $\mathcal{T C}$ and $\alpha$.

3. Suppose that

$$
\begin{aligned}
\mathcal{T C}= & \sum\left\{o ; \mathcal{T C}_{o} \mid o \in A_{O} \cap \text { out }(C)\right\}+ \\
& \sum\left\{o ; \text { fail } \mid o \in A_{O} \backslash \text { out }(C)\right\}+, \\
& \sum\{\sigma ; \text { fail } \mid \sigma \in \Sigma\}
\end{aligned}
$$

with $o \in A_{O}$ and, for all $o \in \operatorname{out}(C), \mathcal{T C}_{o} \in \operatorname{Tests}_{\mathcal{S}}(C$ after $o$ ). Suppose that Lemma 2 holds for all $\mathcal{T} \mathcal{C}_{o}$ and every $\alpha^{\prime} \in \operatorname{testruns}\left(\mathcal{T} \mathcal{C}_{o} \| \mathcal{I}\right)$ for which $\mathcal{T} \mathcal{C}^{\prime} \stackrel{\alpha^{\prime}}{\Rightarrow}$ fail (the induction hypothesis). $\mathcal{T} \mathcal{C}_{o}$ is a subtest of $\mathcal{T C}$. From the shape of $\mathcal{T C}$ it follows that we can distinguish three cases:

(a) if ntrace $(\alpha)=o$ ntrace $\left(\alpha^{\prime}\right)$, with $o \in A_{O} \cap \operatorname{out}(C)$, and $\alpha^{\prime} \in$ testruns $\left(\mathcal{T} \mathcal{C}_{o} \| \mathcal{I}\right)$; then since $\mathcal{T C} \stackrel{o}{\rightarrow} \mathcal{T} \mathcal{C}_{o}$ and (by the induction hypothesis), Lemma 2 holds for $\mathcal{T} \mathcal{C}_{o}$ and $\alpha^{\prime}$, it follows that Lemma 2 holds for $\mathcal{T C}$ and $\alpha$;

(b) if $\operatorname{ntrace}(\alpha)=o$, with $o \in A_{O} \backslash \operatorname{out}(C)$, then, since $\mathcal{T C} \stackrel{\epsilon}{\Rightarrow} \mathcal{T C}$ and $\mathcal{T C} \stackrel{o}{\rightarrow}$ fail it follows that case 1 of Lemma 2 holds for $\mathcal{T C}$ and $\alpha$; or

(c) if $\operatorname{ntrace}(\alpha)=\sigma$, then, since $\mathcal{T C} \stackrel{\epsilon}{\Rightarrow} \mathcal{T C}$ and $\mathcal{T C} \stackrel{\sigma}{\rightsquigarrow}$ fail, it follows that case 2 of Lemma 2 holds for $\mathcal{T C}$ and $\alpha$.

4. Suppose that

$$
\begin{aligned}
& \mathcal{T C}= \sum\left\{\sigma ; \mathcal{T C}_{\sigma} \mid \sigma \in \operatorname{traj}_{\mathbf{u}}(C)\right\}+\sum\left\{\sigma ; \text { fail } \mid \sigma \notin \operatorname{subtraj}_{\mathbf{u}}(C)\right\}+ \\
& \sum\left\{o ; \mathcal{T C}_{o} \mid \sigma \in A_{O} \cap \text { out }(C)\right\}+\sum\left\{o ; \text { fail } \mid o \in A_{O} \backslash \text { out }(C)\right\}+ \\
& \sum\left\{\sigma^{\prime} ; o ; \mathcal{T C}_{\sigma^{\prime} o} \mid \sigma^{\prime} \in \operatorname{subtraj} \mathbf{u}(C) \wedge \sigma^{\prime} . l t i m e<j \wedge\right. \\
& o\left.\in \operatorname{out}\left(C \text { after } \sigma^{\prime}\right)\right\}+ \\
& \sum\left\{\sigma^{\prime} ; o ; \text { fail } \mid \sigma^{\prime} \in \operatorname{subtraj}_{\mathbf{u}}(C) \wedge \sigma^{\prime} . l t i m e<j \wedge\right. \\
&\left.o \notin \operatorname{out}\left(C \text { after } \sigma^{\prime}\right)\right\},
\end{aligned}
$$

$\mathcal{T} \mathcal{C}_{o}, \mathcal{T C}_{\sigma}$, and $\mathcal{T} \mathcal{C}_{\sigma^{\prime} o}$ are subtests of $\mathcal{T C}$. Suppose that Lemma 2 holds for every $\mathcal{T} \mathcal{C}_{\sigma}, \mathcal{T C}_{o}$, and $\mathcal{T} \mathcal{C}_{\sigma o}$ and every $\alpha^{\prime} \in$ testruns $\left(\mathcal{T} \mathcal{C}_{\sigma} \| \mathcal{I}\right) \cup$ testruns $\left(\mathcal{T C}_{o} \|\right.$ $\mathcal{I}) \cup$ testruns $\left(\mathcal{T} \mathcal{C}_{\sigma o} \| \mathcal{I}\right.$ ) (the induction hypothesis). From the shape of $\mathcal{T C}$ it follows that we can distinguish six cases: 
(a) if $\mathbf{n t r a c e}(\alpha)=\sigma \operatorname{ntrace}\left(\alpha^{\prime}\right)$, with $\sigma \in \operatorname{traj}_{\mathbf{u}}(C)$ and $\alpha^{\prime} \in \operatorname{testruns}\left(\mathcal{T C}_{\sigma} \|\right.$ $\mathcal{I})$, then:

i. if $\mathbf{n t r a c e}\left(\alpha^{\prime}\right)=\sigma^{\prime \prime}$, then $\operatorname{ntrace}(\alpha)=\sigma \frown \sigma^{\prime \prime}$ and, since $\mathcal{T C} \stackrel{\epsilon}{\Rightarrow} \mathcal{T}$ and $\mathcal{T C} \stackrel{\sigma}{\rightsquigarrow}$ fail, it follows that case 2 of Lemma 2 holds for $\mathcal{T C}$ and $\alpha$.

ii. if $\mathbf{n t r a c e}\left(\alpha^{\prime}\right)=\sigma^{\prime \prime} o$, then $\operatorname{ntrace}(\alpha)=\sigma \frown \sigma^{\prime \prime} o$ and, since $\mathcal{T C} \stackrel{\epsilon}{\Rightarrow}$ $\mathcal{T C}$ and $\mathcal{T C} \stackrel{\sigma \frown \sigma^{\prime \prime} o}{\Longrightarrow}$ fail, it follows that case 3 of Lemma 2 holds for $\mathcal{T C}$ and $\alpha$; and

iii. in any other case (if ntrace $\left(\alpha^{\prime}\right)=o \alpha^{\prime \prime}$, or ntrace $\left(\alpha^{\prime}\right)=\sigma^{\prime \prime} \alpha^{\prime \prime}$ and $\left.\alpha^{\prime \prime} \neq \epsilon\right)$, since $\mathcal{T C} \stackrel{\sigma}{\rightsquigarrow} \mathcal{T} \mathcal{C}_{\sigma}$ and, by the induction hypotheses, Lemma 2 holds for $\mathcal{T} \mathcal{C}_{\sigma}$ and $\alpha^{\prime}$, it follows that Lemma 2 holds for $\mathcal{T C}$ and $\alpha$;

(b) if $\mathbf{n t r a c e}(\alpha)=\sigma$, with $\sigma \notin \operatorname{subtraj}_{\mathbf{u}}(C)$, then, since $\mathcal{T C} \stackrel{\epsilon}{\Rightarrow} \mathcal{T C}$ and $\mathcal{T C} \stackrel{\sigma}{\rightsquigarrow}$ fail, it follows that case 2 of Lemma 2 holds for $\mathcal{T C}$ and $\alpha$;

(c) if $\operatorname{ntrace}(\alpha)=o$ ntrace $\left(\alpha^{\prime}\right)$, with $o \in A_{O} \cap \operatorname{out}(C)$ and $\alpha^{\prime} \in$ testruns $\left(\mathcal{T} \mathcal{C}_{o} \| \mathcal{I}\right)$; then since $\mathcal{T C} \stackrel{o}{\rightarrow} \mathcal{T} \mathcal{C}_{o}$ and (by the induction hypothesis), Lemma 2 holds for $\mathcal{T} \mathcal{C}_{o}$ and $\alpha^{\prime}$, it follows that Lemma 2 holds for $\mathcal{T C}$ and $\alpha$;

(d) if $\operatorname{ntrace}(\alpha)=o$, with $o \in A_{O} \backslash$ out $(C)$, then, since $\mathcal{T C} \stackrel{\epsilon}{\Rightarrow} \mathcal{T C}$ and $\mathcal{T C} \stackrel{o}{\rightarrow}$ fail it follows that case 1 of Lemma 2 holds for $\mathcal{T C}$ and $\alpha$;

(e) if $\operatorname{ntrace}(\alpha)=\sigma^{\prime} o$ ntrace $\left(\alpha^{\prime}\right)$ with $\sigma^{\prime} \in \operatorname{subtraj}_{\mathbf{u}}(C), o \in \operatorname{out}\left(C \operatorname{after} \sigma^{\prime}\right)$ and $\alpha^{\prime} \in \operatorname{testruns}\left(\mathcal{T} \mathcal{C}_{\sigma^{\prime} o} \| \mathcal{I}\right)$, then, since $\mathcal{T C} \stackrel{\sigma^{\prime} o}{\Rightarrow} \mathcal{T} \mathcal{C}_{\sigma^{\prime} o}$ and, by the induction hypothesis, Lemma 2 holds for $\mathcal{T C}_{\sigma^{\prime} o}$ and $\alpha^{\prime}$, it follows that Lemma 2 holds for $\mathcal{T C}$ and $\alpha$; and

(f) if $\operatorname{ntrace}(\alpha)=\sigma^{\prime} o$, with $\sigma^{\prime} \in \operatorname{subtraj}_{\mathbf{u}}(C)$ and $o \notin \operatorname{out}\left(C\right.$ after $\left.\sigma^{\prime}\right)$, then , since $\mathcal{T C} \stackrel{\epsilon}{\Rightarrow} \mathcal{T C}$ and $\mathcal{T C} \stackrel{\sigma^{\prime} o}{\Rightarrow} \mathcal{T C} \mathcal{C}_{\sigma^{\prime} o}$, it follows that case 2 of Lemma 2 holds for $\mathcal{T C}$ and $\alpha$.

Lemma 3. If $\mathcal{T C} \in \operatorname{Tests}_{\mathcal{S}}\left(\left\{s_{0}\right\}\right)$ and $\alpha \in \operatorname{testruns}(\mathcal{T C} \| \mathcal{I})$ such that $\mathcal{T C} \stackrel{\alpha}{\Rightarrow}$ fail, then:

- if ntrace $(\alpha)=\alpha^{\prime} o$, then $o \notin \operatorname{out}\left(\mathcal{S}\right.$ after $\left.\alpha^{\prime}\right) ;$ and

- if $\operatorname{ntrace}(\alpha)=\alpha^{\prime} \sigma$, then $\sigma \notin \operatorname{traj}\left(\mathcal{S}\right.$ after $\left.\alpha^{\prime}\right)$.

Proof.

- Suppose that ntrace $(\alpha)=\alpha^{\prime}$ o. According to Lemma 2, either:

1. ntrace $(\alpha)=\alpha^{\prime} o$, and there exists a subtest $\mathcal{T} \mathcal{C}^{\prime} \in \operatorname{Tests}_{\mathcal{S}}\left(\mathcal{S}\right.$ after $\left.\alpha^{\prime}\right)$ such that $\mathcal{T C} \stackrel{\alpha^{\prime}}{\Rightarrow} \mathcal{T} \mathcal{C}^{\prime}$ and $\mathcal{T} \mathcal{C}^{\prime} \stackrel{o}{\rightarrow}$ fail; or

2. ntrace $(\alpha)=\alpha^{\prime} \sigma o$, and there exists a subtest $\mathcal{T} \mathcal{C}^{\prime} \in \operatorname{Tests}_{\mathcal{S}}\left(\mathcal{S}\right.$ after $\left.\alpha^{\prime}\right)$ such that $\mathcal{T C} \stackrel{\alpha^{\prime}}{\Rightarrow} \mathcal{T} \mathcal{C}^{\prime}$ and $\mathcal{T} \mathcal{C}^{\prime} \stackrel{\sigma o}{\Rightarrow}$ fail.

Then,

1. By definition of $\operatorname{Tests}_{\mathcal{S}}\left(\mathcal{S}\right.$ after $\left.\alpha^{\prime}\right)$, we know that $\mathcal{T} \mathcal{C}^{\prime}$ is described by either case 3 or case 4 of our test definition. By this definition $\mathcal{T C}^{\prime} \stackrel{o}{\rightarrow}$ fail, only if $o \notin \operatorname{out}\left(\mathcal{S}\right.$ after $\left.\alpha^{\prime}\right)$. 
2. Suppose that $\mathcal{T} \mathcal{C}^{\prime \prime} \in \operatorname{Tests}_{\mathcal{S}}\left(\mathcal{S}\right.$ after $\left.\alpha^{\prime \prime}\right)$ is the last subtest of $\mathcal{T C}$ for trace $\alpha$. That is, supposing ntrace $(\alpha)=\operatorname{ntrace}\left(\alpha^{\prime \prime} \sigma^{\prime \prime} o\right), \mathcal{T C} \stackrel{\alpha^{\prime \prime}}{\Rightarrow} \mathcal{T} \mathcal{C}^{\prime \prime}$ and $\mathcal{T C}^{\prime \prime} \stackrel{\sigma^{\prime \prime} o}{\Rightarrow}$ fail and there does not exist a subtest $\mathcal{T} \mathcal{C}^{\prime \prime \prime}$ such that $\sigma^{\prime \prime}=\sigma_{1}^{\prime \prime} \frown \sigma_{2}^{\prime \prime}$ and $\mathcal{T} \mathcal{C}^{\prime \prime} \stackrel{\sigma_{1}^{\prime \prime}}{\Rightarrow} \mathcal{T} \mathcal{C}^{\prime \prime \prime}$ and $\mathcal{T} \mathcal{C}^{\prime \prime \prime} \stackrel{\sigma_{2}^{\prime \prime} o}{\Rightarrow}$ fail. We know that there exists such $\mathcal{T}^{\prime \prime}$ (since, according to Lemma 2, $\mathcal{T} \mathcal{C}^{\prime}$ could be this last subtest). In this case we know that $\mathcal{T} \mathcal{C}^{\prime \prime}$ is described by case 4 of our test definition and $\sigma^{\prime \prime} \in \operatorname{subtraj}_{\mathbf{u}}\left(S\right.$ after $\left.\alpha^{\prime \prime}\right)$ and $o \notin \operatorname{out}\left(\mathcal{S}\right.$ after $\left.\alpha^{\prime \prime} \sigma^{\prime \prime}\right)$. Because $\alpha^{\prime}=\operatorname{ntrace}\left(\alpha^{\prime \prime} \sigma^{\prime \prime}\right)$ we conclude that $o \notin \operatorname{out}\left(\mathcal{S}\right.$ after $\left.\alpha^{\prime}\right)$

- Suppose that ntrace $(\alpha)=\alpha^{\prime} \sigma$. Suppose that $\mathcal{T C}^{\prime \prime} \in \operatorname{Tests}_{\mathcal{S}}\left(\mathcal{S}\right.$ after $\left.\alpha^{\prime \prime}\right)$ is the last subtest of $\mathcal{T C}$ for trace $\alpha$. That is, supposing ntrace $(\alpha)=$ ntrace $\left(\alpha^{\prime \prime} \sigma^{\prime \prime}\right), \mathcal{T C} \stackrel{\alpha^{\prime \prime}}{\Rightarrow} \mathcal{T} \mathcal{C}^{\prime \prime}$ and $\mathcal{T} \mathcal{C}^{\prime \prime} \stackrel{\sigma^{\prime \prime}}{\rightsquigarrow}$ fail and there does not exists a subtest $\mathcal{T} \mathcal{C}^{\prime \prime \prime}$ such that $\sigma^{\prime \prime}=\sigma_{1}^{\prime \prime} \frown \sigma_{2}^{\prime \prime}$ and $\mathcal{T} \mathcal{C}^{\prime \prime} \stackrel{\sigma_{1}^{\prime \prime}}{\Rightarrow} \mathcal{T} \mathcal{C}^{\prime \prime \prime}$ and $\mathcal{T} \mathcal{C}^{\prime \prime \prime} \stackrel{\sigma_{2}^{\prime \prime}}{\rightsquigarrow}$ fail. Again, we know that there exists such $\mathcal{T C}^{\prime \prime}$ (since, according to Lemma 2, $\mathcal{T C}^{\prime}$ could be this last subtest). In this case $\mathcal{T} \mathcal{C}^{\prime \prime}$ is described by case 3 or case for of our test definition and either $\sigma^{\prime \prime} \notin \Sigma$ or $\sigma^{\prime \prime} \notin \operatorname{subtraj}_{\mathbf{u}}\left(\mathcal{S}\right.$ after $\left.\alpha^{\prime \prime}\right)$, which means $\sigma^{\prime \prime} \notin \operatorname{traj}\left(\mathcal{S}\right.$ after $\left.\alpha^{\prime \prime}\right)$. By condition A2 on our hybrid transition systems we conclude that in this case also $\sigma \notin \operatorname{traj}\left(\mathcal{S}\right.$ after $\left.\alpha^{\prime}\right)$

The following theorem states that if an implementation is conform as specification, all test runs of all tests (according to our inductive definition) lead to verdict pass. In line with other conformance theories we call this soundness of our tests.

Theorem 1. If $\mathcal{S}$ is a specification and $\mathcal{I}$ is an implementation, then:

$$
\mathcal{I} \text { hioco } \mathcal{S} \Longrightarrow \forall_{\mathcal{T C} \in T e s t s_{\mathcal{S}}\left(\left\{s_{0}\right\}\right)}: \mathcal{I} \text { passes } \mathcal{T C} \text {. }
$$

Proof. We suppose that $\mathcal{I}$ hioco $\mathcal{S}$ and $s^{\prime} \in S_{\mathcal{I}}$, and we prove that for all tests that can be derived from $\mathcal{S}$, there does not exist an $\alpha \in \operatorname{testruns}(\mathcal{T C} \| \mathcal{I})$ such that $\left(t_{0}, s_{0}\right) \stackrel{\alpha}{\Rightarrow}$ (fail, $\left.s^{\prime}\right)$. We prove this by contraposition. We suppose that $\mathcal{T C} \in$ $\operatorname{Tests}_{\mathcal{S}}\left(\left\{s_{0}\right\}\right)$ and $\alpha \in \operatorname{testruns}(\mathcal{T C} \| \mathcal{I})$ and $s^{\prime} \in S_{\mathcal{I}}$ and $\left(t_{0}, s_{0}\right) \stackrel{\alpha}{\Rightarrow}$ (fail, $\left.s^{\prime}\right)$, and we show that this $\alpha$ can only exist if $\mathcal{I}$ hioco $\mathcal{S}$.

From Lemma 1 and by definition of ntrace $(\alpha)$ we know that for every $\mathcal{T C} \stackrel{\alpha}{\Rightarrow}$ fail there exists an $\alpha^{\prime}$ and $o$ and $\sigma$ such that either ntrace $(\alpha)=\alpha^{\prime} o$, ntrace $(\alpha)=\alpha^{\prime} \sigma$. Because a test is strongly time deterministic we know that if $\mathcal{T C} \stackrel{\alpha}{\Rightarrow}$ fail then $\mathcal{T C} \stackrel{\text { ntrace }(\alpha)}{\Longrightarrow}$ fail.

- Suppose that ntrace $(\alpha)=\alpha^{\prime} o$. From Lemma 3 we know that $o \notin \operatorname{out}\left(\mathcal{S}\right.$ after $\left.\alpha^{\prime}\right)$. Because ntrace $(\alpha) \in \operatorname{testruns}(\mathcal{T C} \| \mathcal{I})$ we also know that $o \in\left(\mathcal{I}\right.$ after $\left.\alpha^{\prime}\right)$ (by definition of synchronous composition). Therefore, out $\left(\mathcal{I}\right.$ after $\left.\alpha^{\prime}\right) \nsubseteq$ out $\left(\mathcal{S}\right.$ after $\left.\alpha^{\prime}\right)$.

- Suppose that ntrace $(\alpha)=\alpha^{\prime} \sigma$. From Lemma 3 we know that $\sigma \notin \operatorname{traj}\left(\mathcal{S}\right.$ after $\left.\alpha^{\prime}\right)$. Because ntrace $(\alpha) \in \operatorname{testruns}(\mathcal{T C} \| \mathcal{I})$ we also know that $\sigma \in\left(\mathcal{I}\right.$ after $\left.\alpha^{\prime}\right)$ 
(by definition of synchronous composition). From Lemma 2 we know there exists a subtest $\mathcal{T} \mathcal{C}^{\prime} \in \operatorname{Tests}_{\mathcal{S}}\left(\mathcal{S}\right.$ after $\left.\alpha^{\prime}\right)$ such that $\mathcal{T C} \stackrel{\alpha^{\prime}}{\Rightarrow} \mathcal{T} \mathcal{C}^{\prime}$ and $\mathcal{T C} \stackrel{\sigma}{\rightsquigarrow}$ fail.

- Suppose that $\mathcal{T} \mathcal{C}^{\prime}$ is a test described by case 3 of the definition of $\operatorname{Tests}_{\mathcal{S}}\left(\mathcal{S}\right.$ after $\left.\alpha^{\prime}\right)$. Then, $\operatorname{traj}\left(\mathcal{S}\right.$ after $\left.\alpha^{\prime}\right)=\emptyset$ which means $\xi \notin$ $\operatorname{out}\left(S\right.$ after $\left.\alpha^{\prime}\right)$. However, $\operatorname{since} \operatorname{traj}\left(\mathcal{I}\right.$ after $\left.\alpha^{\prime}\right) \neq \emptyset, \xi \in \operatorname{out}\left(\mathcal{I}\right.$ after $\left.\alpha^{\prime}\right)$. Therefore, out $\left(\mathcal{I}\right.$ after $\left.\alpha^{\prime}\right) \nsubseteq \mathbb{\operatorname { o u t }}\left(\mathcal{S}\right.$ after $\left.\alpha^{\prime}\right)$.

- Suppose that $\mathcal{T} \mathcal{C}^{\prime}$ is a test described by case 4 of the definition of $\operatorname{Tests}_{\mathcal{S}}\left(\mathcal{S}\right.$ after $\left.\alpha^{\prime}\right)$. Because, by the definition of tests, the input behavior is always selected from the specification $\sigma \in \operatorname{infilter}\left(\operatorname{traj}\left(\mathcal{I}\right.\right.$ after $\left.\alpha^{\prime}\right)$, $\operatorname{traj}\left(\mathcal{S}\right.$ after $\left.\left.\alpha^{\prime}\right)\right)$. Therefore, $\operatorname{infilter}\left(\operatorname{traj}(\mathcal{I}\right.$ after $\alpha), \operatorname{traj}\left(\mathcal{S}\right.$ after $\left.\left.\alpha^{\prime}\right)\right) \nsubseteq$ $\operatorname{traj}\left(\mathcal{S}\right.$ after $\left.\alpha^{\prime}\right)$.

We conclude that, if $\alpha \in$ testruns $(\mathcal{T C} \| \mathcal{I})$ and $\mathcal{T C} \stackrel{\alpha}{\Rightarrow}$ fail, then $\mathcal{I}$ hioco $\mathcal{S}$. This contradicts the assumption that $\mathcal{I}$ hioco $\mathcal{S}$.

The following theorem states that if an implementation is not conform a specification $(\mathcal{I}$ hioco $\mathcal{S}$ ), then there is a test $\mathcal{T C}$ which leads to verdict fail $(\mathcal{I}$ passes $\mathcal{T C})$. In line with the ioco theory we call this exhaustiveness of our tests.

Theorem 2. If $\mathcal{S}$ is a specification and $\mathcal{I}$ is an implementation, then:

$\mathcal{I}$ hioco $\mathcal{S} \Longrightarrow \exists_{\mathcal{T C} \in T e s t s_{\mathcal{S}}\left(\left\{s_{0}\right\}\right)}: \mathcal{I}$ passes $\mathcal{T C}$.

Proof. Let $C_{\mathcal{I}} \subseteq S_{\mathcal{I}}$ and $C_{\mathcal{S}} \subseteq S_{\mathcal{S}}$. We prove that if

$$
\begin{aligned}
& \operatorname{out}\left(C_{\mathcal{I}} \operatorname{after} \alpha\right) \nsubseteq \operatorname{out}\left(C_{\mathcal{S}} \text { after } \alpha\right) \vee \\
& \operatorname{infilter}\left(\operatorname{traj}\left(C_{\mathcal{I}} \text { after } \alpha\right), \operatorname{traj}\left(C_{\mathcal{S}} \text { after } \alpha\right)\right) \nsubseteq \operatorname{traj}\left(C_{\mathcal{S}} \text { after } \alpha\right)
\end{aligned}
$$

then there exists a $\mathcal{T C} \in \operatorname{Tests}_{\mathcal{S}}\left(C_{\mathcal{S}}\right)$ with initial state $t_{0}$ and $\alpha \mu \in$ testruns $(\mathcal{T C} \|$ $\mathcal{I}$ ) and, with $c \in C_{\mathcal{I}}$ and $c^{\prime} \in C$ after $\alpha \mu,\left(t_{0}, c\right) \stackrel{\alpha \mu}{\Rightarrow}$ (fail, $c^{\prime}$ ). Then, the theorem follows for $C_{\mathcal{I}}=\left\{s_{0 \mathcal{I}}\right\}$ and $C_{\mathcal{S}}=\left\{s_{0 \mathcal{S}}\right\}$. We proceed by induction on the length of $\alpha$.

- Suppose that $\alpha=\epsilon$. Then,

$$
\operatorname{out}\left(C_{\mathcal{I}}\right) \nsubseteq \operatorname{out}\left(C_{\mathcal{S}}\right) \vee \operatorname{infilter}\left(\operatorname{traj}\left(C_{\mathcal{I}}\right), \operatorname{traj}\left(C_{\mathcal{S}}\right)\right) \nsubseteq \operatorname{traj}\left(C_{\mathcal{S}}\right) .
$$

- Suppose that there exists an $o \in \operatorname{out}\left(C_{\mathcal{I}}\right) \backslash \operatorname{out}\left(C_{\mathcal{S}}\right)$.

* Suppose that $\operatorname{traj}\left(C_{\mathcal{I}}\right)=\emptyset$ Then, for instance, $\mathcal{T C}=$

$$
\begin{aligned}
& \sum\left\{o ; \text { pass } \mid o \in A_{O} \cap \operatorname{out}\left(C_{\mathcal{S}}\right)\right\}+ \\
& \sum\left\{o ; \text { fail } \mid o \in A_{O} \backslash \text { out }\left(C_{\mathcal{S}}\right)\right\}+ \\
& \sum\{\sigma ; \text { fail } \mid \sigma \in \Sigma\}
\end{aligned}
$$

leads to verdict fail (since $o \notin$ out $\left(C_{\mathcal{S}}\right)$ ), and therefore $\mathcal{T C} \stackrel{o}{\rightarrow}$ fail. 
* Suppose that $\operatorname{traj}\left(C_{\mathcal{I}}\right)=\emptyset$. Then, for instance, with some $u \in\left\{\sigma \downarrow V_{I} \mid \sigma \in \operatorname{traj}\left(C_{\mathcal{S}}\right)\right\}$ and $j=u . l t i m e, \mathcal{T C}=$

$$
\begin{aligned}
& \sum\left\{\sigma ; \mathbf{p a s s} \mid \sigma \in \operatorname{traj}_{\mathbf{u}}\left(C_{\mathcal{S}}\right)\right\}+\sum\left\{\sigma ; \text { fail } \mid \sigma \notin \text { subtraj }_{\mathbf{u}}\left(C_{\mathcal{S}}\right)\right\}+ \\
& \sum\left\{o ; \text { pass } \mid o \in A_{O} \cap \operatorname{out}\left(C_{\mathcal{S}}\right)\right\}+\sum\left\{o ; \text { fail } \mid o \in A_{O} \backslash \operatorname{out}\left(C_{\mathcal{S}}\right)\right\}+ \\
& \sum\left\{\sigma^{\prime} ; o ; \text { pass } \mid \sigma^{\prime} \in \text { subtraju }_{\mathbf{u}}\left(C_{\mathcal{S}}\right) \wedge \sigma^{\prime} \text {.ltime }<j \wedge\right. \\
& \sum\left\{\sigma^{\prime} ; o ; \text { fail } \mid \sigma^{\prime} \in \operatorname{subtraj}_{\mathbf{u}}\left(C_{\mathcal{S}}\right) \wedge \sigma^{\prime} . \text { ltime }<j \wedge\right. \\
& \left.o \in \operatorname{out}\left(C_{\mathcal{S}} \text { after } \sigma^{\prime}\right)\right\}+ \\
& \left.o \notin \operatorname{out}\left(C_{\mathcal{S}} \text { after } \sigma^{\prime}\right)\right\}
\end{aligned}
$$

leads to verdict fail (since $o \notin \operatorname{out}\left(C_{\mathcal{S}}\right)$ ), and therefore $\mathcal{T C} \stackrel{o}{\rightarrow}$ fail. In both cases, since $o \in \operatorname{out}\left(C_{\mathcal{I}}\right),\left(t_{0}^{\prime}, c\right) \stackrel{o}{\Rightarrow}\left(\right.$ fail,$\left.c^{\prime}\right)$.

- Suppose that there exists a $\sigma \in \operatorname{infilter}\left(\operatorname{traj}\left(C_{\mathcal{I}}\right), \operatorname{traj}\left(C_{\mathcal{S}}\right)\right) \backslash \operatorname{traj}\left(C_{\mathcal{S}}\right)$.

Then, for instance, with $u=\sigma \downarrow V_{I}$ and $j=u$.ltime, $\mathcal{T} \mathcal{C}=$

$$
\begin{aligned}
& \sum\left\{\sigma ; \mathbf{p a s s} \mid \sigma \in \operatorname{traj}_{\mathbf{u}}\left(C_{\mathcal{S}}\right)\right\}+\sum\left\{\sigma ; \text { fail } \mid \sigma \notin \text { subtraj }_{\mathbf{u}}\left(C_{\mathcal{S}}\right)\right\}+ \\
& \sum\left\{o ; \operatorname{pass} \mid o \in A_{O} \cap \operatorname{out}\left(C_{\mathcal{S}}\right)\right\}+\sum\left\{o ; \text { fail } \mid o \in A_{O} \backslash \text { out }\left(C_{\mathcal{S}}\right)\right\}+ \\
& \sum\left\{\sigma^{\prime} ; o ; \text { pass } \mid \sigma^{\prime} \in \text { subtraju }_{\mathbf{u}}\left(C_{\mathcal{S}}\right) \wedge \sigma^{\prime} \text {.ltime }<j \wedge\right. \\
& \sum\left\{\sigma^{\prime} ; o ; \text { fail } \mid \sigma^{\prime} \in \text { subtraju }_{\mathbf{u}}\left(C_{\mathcal{S}}\right) \wedge \sigma^{\prime} . \text { ltime }<j \wedge\right. \\
& \left.o \in \operatorname{out}\left(C_{\mathcal{S}} \text { after } \sigma^{\prime}\right)\right\}+ \\
& \left.o \notin \operatorname{out}\left(C_{\mathcal{S}} \text { after } \sigma^{\prime}\right)\right\}
\end{aligned}
$$

leads to verdict fail (since $\sigma \notin \operatorname{traj}_{\mathbf{u}}\left(C_{\mathcal{S}}\right)$ ). Hence, since $\sigma \in \operatorname{traj}\left(C_{\mathcal{I}}\right.$ ), $\left(t_{0}^{\prime}, c\right) \stackrel{\sigma}{\Rightarrow}\left(\right.$ fail,$\left.c^{\prime}\right)$.

- Suppose that $\xi \in \operatorname{out}\left(C_{\mathcal{I}}\right) \backslash \operatorname{out}\left(C_{\mathcal{S}}\right)$. In this case we know that $\operatorname{traj}\left(C_{\mathcal{S}}\right)=$

$\emptyset$. Then, for instance, $\mathcal{T C}=$

$$
\begin{aligned}
& \sum\left\{o ; \text { pass } \mid o \in A_{O} \cap \operatorname{out}\left(C_{\mathcal{S}}\right)\right\}+ \\
& \sum\left\{o ; \text { fail } \mid o \in A_{O} \backslash \text { out }\left(C_{\mathcal{S}}\right)\right\}+ \\
& \sum\{\sigma ; \text { fail } \mid \sigma \in \Sigma\}
\end{aligned}
$$

leads to verdict fail for any $\sigma \in \Sigma, \mathcal{T C} \stackrel{\sigma}{\rightsquigarrow}$ fail. Hence, since $\operatorname{traj}\left(C_{\mathcal{I}}\right) \neq$ $\emptyset$, for any $\sigma \in \operatorname{traj}\left(C_{\mathcal{I}}\right),\left(t_{0}^{\prime}, c\right) \stackrel{\sigma}{\Rightarrow}\left(\right.$ fail,$\left.c^{\prime}\right)$.

- Suppose that $\alpha=\nu \alpha^{\prime}$ and (the induction hypothesis):

$$
\begin{aligned}
& \operatorname{out}\left(\left(C_{\mathcal{I}} \text { after } \nu\right) \text { after } \alpha^{\prime}\right) \nsubseteq \operatorname{out}\left(\left(C_{\mathcal{S}} \text { after } \nu\right) \text { after } \alpha^{\prime}\right) \vee \\
& \operatorname{infilter}\left(\operatorname{traj}\left(\left(C_{\mathcal{I}} \text { after } \nu\right) \text { after } \alpha^{\prime}\right), \operatorname{traj}\left(\left(C_{\mathcal{S}} \text { after } \nu\right) \text { after } \alpha^{\prime}\right)\right) \nsubseteq \\
& \quad \operatorname{traj}\left(\left(C_{\mathcal{S}} \text { after } \nu\right) \text { after } \alpha^{\prime}\right)
\end{aligned}
$$

and there exists a $\mathcal{T C} \in \operatorname{Tests}_{\mathcal{S}}\left(C_{\mathcal{S}}\right.$ after $\left.\nu\right)$ with initial state $t_{0}$ and $\alpha \mu \in$ testruns $(\mathcal{T C} \| \mathcal{I})$ and, with $c \in C_{\mathcal{I}}$ and $c^{\prime} \in C_{\mathcal{I}}$ after $\alpha \mu,\left(t_{0}, c\right) \stackrel{\alpha \mu}{\Rightarrow}$ (fail, $\left.c^{\prime}\right)$.

- Suppose that $\nu=i$, with $i \in A_{I}$. Then, the test $i ; \mathcal{T} \mathcal{C}$ will also lead to verdict fail for trace $\alpha$.

- Suppose $\nu=o$, with $o \in A_{O} \cap \operatorname{out}\left(C_{\mathcal{S}}\right)$, and $\operatorname{traj}(\mathcal{S})=\emptyset$. Then, for instance, the test

$$
\begin{aligned}
& \sum\left\{o^{\prime} ; \mathcal{T C}_{o^{\prime}} \mid o^{\prime} \in A_{O} \cap \operatorname{out}\left(C_{\mathcal{S}}\right)\right\}+ \\
& \sum\left\{o^{\prime} ; \text { fail } \mid o^{\prime} \in A_{O} \backslash \text { out }\left(C_{\mathcal{S}}\right)\right\}+ \\
& \sum\{\sigma ; \text { fail } \mid \sigma \in \Sigma\}
\end{aligned}
$$


with $\mathcal{T C}_{o}=\mathcal{T C}$ and, for $o^{\prime} \in\left(A_{O} \cap \operatorname{out}\left(C_{\mathcal{S}}\right)\right) \backslash\{o\}, t c_{o}^{\prime}=$ pass will also lead to verdict fail for trace $\alpha$.

- Suppose that $\nu=o$, with $o \in A_{O} \cap \operatorname{out}\left(C_{\mathcal{S}}\right)$, and $\operatorname{traj}(\mathcal{S}) \neq \emptyset$. Then, for instance, the test

$$
\begin{gathered}
\sum\left\{\sigma^{\prime} ; \text { pass } \mid \sigma^{\prime} \in \text { traj }_{\mathbf{u}}\left(C_{\mathcal{S}}\right)\right\}+\sum\left\{\sigma^{\prime} ; \text { fail } \mid \sigma^{\prime} \notin \text { subtraj }_{\mathbf{u}}\left(C_{\mathcal{S}}\right)\right\}+ \\
\sum\left\{o^{\prime} ; \mathcal{T C}_{o^{\prime}} \mid o^{\prime} \in A_{O} \cap \text { out }\left(C_{\mathcal{S}}\right)\right\}+\sum\left\{o^{\prime} ; \text { fail } \mid o^{\prime} \in A_{O} \backslash \text { out }\left(C_{\mathcal{S}}\right)\right\}+ \\
\sum\left\{\sigma^{\prime} ; o ; \text { pass } \mid \sigma^{\prime} \in \operatorname{subtraj}_{\mathbf{u}}\left(C_{\mathcal{S}}\right) \wedge \sigma^{\prime} . l t i m e<j\right. \\
\left.\quad o \in \operatorname{out}\left(C_{\mathcal{S}} \text { after } \sigma^{\prime}\right)\right\}+ \\
\sum\left\{\sigma^{\prime} ; o ; \text { pass } \mid \sigma^{\prime} \in \text { subtraj ju }_{\mathbf{u}}\left(C_{\mathcal{S}}\right) \wedge \sigma^{\prime} . \text { ltime }<j \wedge\right. \\
\left.o \notin \operatorname{out}\left(C_{\mathcal{S}} \text { after } \sigma^{\prime}\right)\right\}
\end{gathered}
$$

with, for some $\sigma^{\prime} \in \operatorname{traj}\left(C_{\mathcal{S}}\right), u=\sigma^{\prime} \downarrow V_{I}$ and $j=u$.ltime and $\mathcal{T C}_{o}=$ $\mathcal{T C}$ and, for $o^{\prime} \in\left(A_{O} \cap \operatorname{out}\left(C_{\mathcal{S}}\right)\right) \backslash\{o\}, t c_{o}^{\prime}=$ pass will also lead to verdict fail for trace $\alpha$. And,

- Suppose that $\nu=\sigma$, with $\sigma \in \operatorname{traj}\left(C_{\mathcal{S}}\right)$. Then, for instance, the test

$$
\begin{gathered}
\sum\left\{\sigma^{\prime} ; \mathcal{T C}_{\sigma^{\prime}} \mid \sigma^{\prime} \in \operatorname{traj}_{\mathbf{u}}\left(C_{\mathcal{S}}\right)\right\}+\sum\left\{\sigma^{\prime} ; \text { fail } \mid \sigma \notin \text { subtraj }_{\mathbf{u}}\left(C_{\mathcal{S}}\right)\right\}+ \\
\sum\left\{o ; \text { pass } \mid o \in A_{O} \cap \text { out }\left(C_{\mathcal{S}}\right)\right\}+\sum\left\{o ; \text { fail } \mid o \in A_{O} \backslash \text { out }\left(C_{\mathcal{S}}\right)\right\}+ \\
\sum\left\{\sigma^{\prime} ; o ; \text { pass } \mid \sigma^{\prime} \in \operatorname{subtraj} \mathbf{u}\left(C_{\mathcal{S}}\right) \wedge \sigma^{\prime} . \text { ltime }<j \wedge\right. \\
\left.o \in \operatorname{out}\left(C_{\mathcal{S}} \text { after } \sigma^{\prime}\right)\right\}+ \\
\sum\left\{\sigma^{\prime} ; o ; \text { fail } \mid \sigma^{\prime} \in \text { subtraj }_{\mathbf{u}}\left(C_{\mathcal{S}}\right) \wedge \sigma^{\prime} . \text { ltime }<j \wedge\right. \\
\left.o \notin \operatorname{out}\left(C_{\mathcal{S}} \text { after } \sigma^{\prime}\right)\right\}
\end{gathered}
$$

with $u=\sigma \downarrow V_{I}$ and $j=$ o.ltime and $\mathcal{T} \mathcal{C}_{\sigma}=\mathcal{T C}$ and, for $\sigma^{\prime} \in$ $\operatorname{traj}\left(C_{\mathcal{S}}\right) \backslash\{\sigma\}, \mathcal{T C}_{\sigma^{\prime}}=$ pass will also lead to verdict fail for trace $\alpha$.

\section{Towards Hybrid Model-based Testing in Practice}

The next step in our research will be to develop tooling based on our hybrid test theory. As a first step we reformulate the inductive definition of tests in section 4 as a recursive test generation algorithm. Let $\mathcal{S}$ be a specification and let $C$ be a set of initial states of $\mathcal{S}$; then tests are generated by the following algorithm.

algorithm $\operatorname{tcg}(\mathcal{S}, C)=$

select non-deterministically

1. $\mathcal{T C}:=$ pass

2. select an $i \in\left\{a \mid a \in A_{I} \wedge C\right.$ after $\left.a \neq \emptyset\right\}$

$\mathcal{T C}:=i ; \operatorname{tcg}(\mathcal{S}, C$ after $i)$

3. if $\operatorname{traj}(C)=\emptyset$ and there exists an $o \in A_{O}$ such that $C$ after $o \neq \emptyset$, then

$$
\begin{aligned}
\mathcal{T C}:= & \sum\left\{o ; \operatorname{tcg}(\mathcal{S}, C \text { after } o) \mid o \in A_{o} \cap \operatorname{out}(C)\right\}+ \\
& \sum\left\{o ; \text { fail } \mid o \in A_{o} \backslash \text { out }(C)\right\}+ \\
& \sum\left\{\sigma ; \text { fail } \mid \sigma \in \Sigma_{\mathcal{S}}\right\}
\end{aligned}
$$

4. if $\operatorname{traj}(C) \neq \emptyset$ then

select an $u \in\left\{\sigma \downarrow V_{I} \mid \sigma \in \operatorname{traj}(C)\right\}$ 


$$
\begin{aligned}
& \text { let } \operatorname{traj}_{\mathbf{u}}(C)=\left\{\sigma \mid \sigma \downarrow V_{I}=u \wedge \sigma \in \operatorname{traj}(C)\right\} \\
& \text { let } \text { subtraju}_{\mathbf{u}}(C)=\left\{\sigma \mid \exists_{\sigma^{\prime} \in \operatorname{traj}_{\mathbf{u}}(C)}: \sigma \leq \sigma^{\prime}\right\} \\
& \mathcal{T C}:=\sum\left\{\sigma ; \operatorname{tcg}(\mathcal{S}, C \text { after } \sigma) \mid \sigma \in \operatorname{traj}_{\mathbf{u}}(C)\right\}+ \\
& \sum\left\{\sigma ; \text { fail } \mid \sigma \notin \text { subtraj }_{\mathbf{u}}(C)\right\}+ \\
& \sum\left\{o ; \mathcal{T C}_{o} \mid o \in A_{O} \cap \operatorname{out}(C)\right\}+\sum\left\{o ; \text { fail } \mid o \in A_{O} \backslash \text { out }(C)\right\}+ \\
& \sum\left\{\sigma^{\prime} ; o ; \operatorname{tcg}\left(\mathcal{S}, C \text { after } \sigma^{\prime} o\right) \mid \sigma^{\prime} \in \operatorname{subtraj}_{\mathbf{u}}(C) \wedge\right. \\
& \left.\sigma^{\prime} . \text { ltime }<u \text {.ltime } \wedge o \in \text { out }\left(C \text { after } \sigma^{\prime}\right)\right\}+ \\
& \sum\left\{\sigma^{\prime} ; o ; \text { fail } \mid \sigma^{\prime} \in \operatorname{subtraj}_{\mathbf{u}}(C) \wedge\right. \\
& \left.\sigma^{\prime} \text {.ltime }<u \text {.ltime } \wedge o \notin \operatorname{out}\left(C \text { after } \sigma^{\prime}\right)\right\}
\end{aligned}
$$

return $\mathcal{T C}$

This algorithm is not directly implementable. Below we discuss several implementation issues that need to be resolved.

In our theory and our algorithm a test has uncountable many states and trajectories and summations over infinite domains. In practice we can of course only deal with finite representations for tests: e.g. trajectories specified as differential equations or algebraic equations, or only by specifying on valuations of variables, and sets of states represented symbolically: e.g. by zones. We prefer to describe tests in the same language as the specification, which we also want to use for simulation and verification. In practice for instance Charon [1], PHAVer [?], or hybrid $\chi[12]$ can be suitable languages.

In our algorithm we did not define how we select input actions and input trajectories. In practice we need to select and generate possible input trajectories in some way. A first way is to let the test generator choose input actions and trajectories at random. A second way is to have the user of the test tool manually select input and construct tests interactively with the tool. A third way is to do guided input selection based on coverage criteria or based on the (physical) behavior of the environment of the system under test.

In our theory continuous behavior is defined over a dense real time domain. In practice tests can only be executed by stimulating the implementation with samples of input, and by observing samples of output in small time steps. In this case selecting a trajectory is selecting a sequence of samples. We can only conclude a verdict with respect to the samples we observe. If according to our specification we need to observe a constant flow of 0 , then it is theoretically possible that we observe value 0 in all samples, while in between samples the value fluctuates. Furthermore, samples will be rounded and therefore we can only conclude a verdict with respect to rounded observations.

In our theory the behavior of a test with respect to an implementation is defined by the synchronous composition of a test and an implementation. In practice we need to stimulate the implementation with input generated from the test and we need to observe the output from the implementation. The first option is to generate executable code from a test. This program provides input for an implementation and observes the output of the implementation. An in- 
frastructure is needed to connect implementation and test. The second option is on the fly test generation. In this case a test tool selects one input (action or trajectory), computes which verdict to attach to the output, and then provides the input to the implementation. It observes the output from the implementation simultaneously, after which a new input (action or trajectory) is selected and applied (in case the test did not lead to verdict fail). An infrastructure is needed to connect the test tool with the implementation under test. On the fly test generation seems more practical. However it might not be possible to the select input and apply the input and observe the output and give a verdict, all in real-time. We need to investigate which method works (best).

\section{Conclusions}

In this report we presented the hybrid input-output conformance relation hioco for hybrid systems. This relation defines in which cases we consider an hybrid implementation correct with respect to a hybrid specification. We presented an inductive definition of tests that is sound and exhaustive.

Our hybrid conformance relation and test definition are based on the discrete conformance relation of Tretmans $[9,10]$ and his test generation procedure for discrete systems. The differences are, besides including trajectories, that we do not have the quiescence action $\delta$ and that we introduce the symbol $\xi$ to indicate the presence of urgent actions. In our tests we observe this $\xi$ implicitly when an urgent output action did not occur but a trajectory is applied and observed instead (which lead to a fail verdict because the trajectory is not allowed according to the specification).

The real-time conformance relation developed by Brandan [2] is also based the discrete conformance theory of Tretmans. However, we believe that our hybrid conformance relation is closer related to the real-time conformance relation of Krichen [6] because in that relation time is viewed as (continuous) output of the implementation.

The next step in our research is to take our hybrid test theory into practice and develop tooling. We acknowledge that in our theory we allow uncountable many actions, trajectories, states, and uncountable many tests. We also acknowledge that in our theory we allow dense real-time and trajectories are selected, applied and observed in synchrony with each other and without delay. We need to fit our theory to what is possible in practice but we can still relate a practical implementation to the theory.

The only research in hybrid model based testing before our hybrid test theory was a proof of concept tester [8] developed at the University of Pennsylvania. No theory was formed for this test tool. The idea of this tool is that a tester is generated from an environment model (that provides the input) and check temporal properties at run time. This is different from our theory in which we generate tests, containing both input and possible output. The advantage of doing this is that in our case a test also specifies the relation between input and output. With the Charon tester it is only possible to test whether the continuous out- 
put of the implementation stays within certain bounds (e.g. whether a variable ever exceeds value 3). Our theory provides the possibility to test whether the continuous output variables behave according to the specified flow of variables.

\section{Acknowledgments}

Hereby I would like to thank Bas Luttik and Jos Baeten from the Computer Science department of the Technische Universiteit Eindhoven for their help in forming this theory and reviewing this report. Furthermore I would like to thank Jan Tretmans, Tim Willemse and René de Vries of the Radboud Universiteit Nijmegen for their good advices as well.

\section{References}

1. R. Alur, T. Dang, J. Esposito, R. Fierro, Y. Hur, F. Ivancic, V. Kumar amd I. Lee, P. Mishra, G. Pappas, and O. Sokolsky. Hierarchical Hybrid Modeling of Embedded Systems. In EMSOFT'01: First Workshop on Embedded Software, 2001.

2. L. Brandán Briones and Ed Brinksma. A test generation framework for quiescent real-time systems. In J. Grabowski and B. Nielsen, editors, FATES04. Formal Approaches to Testing of Software (4th International Workshop), 2004.

3. P.J.L. Cuijpers, M.A. Reniers, and W.P.M.H. Heemels. Hybrid transition systems. Computer Science Reports 02-12, TU/e Department of Computer Science, December 2002.

4. L. Frantzen, J. Tretmans, and T.A.C. Willemse. Test Generation Based on Symbolic Specifications. In J. Grabowski and B. Nielsen, editors, FATES04. Formal Approaches to Testing of Software (4th International Workshop), 2004.

5. L. Frantzen, J. Tretmans, and T.A.C. Willemse. A symbolic framework for modelbased testing. In K. Havelund, M. Núñez, G. Rosu, and B. Wolff, editors, FATES 2006, LNCS. Springer, 2006.

6. M. Kirchen and S. Tripakis. Black-box Conformance Testing for Real-time Systems. In SPIN 2004, pages 109-126. Springer-Verlag, 2004.

7. K. Larsen, M. Mikucionis, and B. Nielsen. Online Testing of Real-time Systems. In J. Grabowski and B. Nielsen, editors, FATES04. Formal Approaches to Testing of Software (4th International Workshop), 2004.

8. L. Tan, O. Sokolsky, , and I. Lee. Specification-based Testing with Linear Temporal Logic. In IEEE Internation Conference on Information Reuse and Integration (IRI'04), 2004.

9. J. Tretmans. Test Generation with Inputs, Outputs and Repetitive Quiescence. Software-Concepts and Tools, 17(3):103-120, 1996.

10. J. Tretmans. Testing concurrent systems: A formal approach. In J.C.M Baeten and S. Mauw, editors, CONCUR'g9 - $10^{\text {th }}$ Int. Conference on Concurrency Theory, volume 1664 of Lecture Notes in Computer Science, pages 46-65. Springer-Verlag, 1999.

11. J. Tretmans and E. Brinksma. TorX: Automated Model Based Testing. In A. Hartman and K. Dussa-Ziegler, editors, Proceedings of the 1st European Conference on Model-Driven Software Engineering, 2003.

12. D.A. van Beek, K.L. Man, M.A. Reniers, J.E. Rooda, and R.R.H. Schiffelers. Syntax and Consistent Equation Semantics of Hybrid Chi. Journal of Logic and Algebraic Programming, special issue on hybrid systems, to appear. 